\title{
Symmetries of K3 sigma models
}

\author{
Matthias R. Gaberdiel, Stefan Hohenegger \\ and RoBerto Volpato
}

It is shown that the supersymmetry-preserving automorphisms of any non-linear $\sigma$-model on K3 generate a subgroup of the Conway group $\mathrm{Co}_{1}$. This is the stringy generalization of the classical theorem, due to Mukai and Kondo, showing that the symplectic automorphisms of any K3 manifold form a subgroup of the Mathieu group $\mathbb{M}_{23}$. The Conway group $\mathrm{Co}_{1}$ contains the Mathieu group $\mathbb{M}_{24}$ (and therefore in particular $\mathbb{M}_{23}$ ) as a subgroup. We confirm the predictions of the Theorem with three explicit conformal field theory (CFT) realizations of $\mathrm{K} 3$ : the $\mathbb{T}^{4} / \mathbb{Z}_{2}$ orbifold at a self-dual point, and the two Gepner models $(2)^{4}$ and $(1)^{6}$. In each case we demonstrate that their symmetries do not form a subgroup of $\mathbb{M}_{24}$, but lie inside $\mathrm{Co}_{1}$ as predicted by our Theorem.

1. Introduction

1.2. Comments and outline

2. Symmetries of non-linear $\sigma$-models on K3

2.1. Moduli space of non-linear $\sigma$-models on K3

2.2. Characterization of $\mathcal{N}=(4,4)$ preserving symmetries

2.3. Classification of the groups of symmetries

3. The $\mathbb{Z}_{2}$ orbifold model

3.1. The spectrum in the $R R$ sector

3.2. Symmetries and twining genera

3.3. The D-brane charge lattice 
2 Matthias R. Gaberdiel, Stefan Hohenegger and Roberto Volpato

4. The Gepner model (1) ${ }^{6} \quad 18$

$\begin{array}{ll}\text { 4.1. The spectrum } & 18\end{array}$

4.2. Symmetries and twining genera $\quad 20$

4.3. The D-brane charge lattice 22

5. The $(2)^{4}$ model 24

$\begin{array}{ll}\text { 5.1. The spectrum } & 24\end{array}$

5.2. Symmetries and twining genera $\quad 26$

5.3. The D-brane charge lattice 28

$\begin{array}{ll}\text { 6. Conclusions } & 28\end{array}$

$\begin{array}{ll}\text { Acknowledgments } & 30\end{array}$

Appendix A. Notation and mathematical background $\quad 30$

Appendix A.1. Group theory 30

$\begin{array}{ll}\text { Appendix A.2. Lattices } & 31\end{array}$

Appendix A.3. The Leech lattice and the Golay code 32

Appendix B. Proof of the Theorem 33

Appendix B.1. $G$ as a subgroup of $O\left(\Gamma^{25,1}\right)$

Appendix B.2. $G$ as a subgroup of $\mathrm{Co}_{0}$

Appendix B.3. The proof of the Theorem 36

Appendix B.4. Realizing all symmetry groups of K3 39 
Appendix C.1. $\mathcal{N}=2$ Minimal models and Gepner
models at $c=6$

Appendix C.2. D-branes in Gepner models

\section{References}

\section{Introduction}

Recently, a hidden $\mathbb{M}_{24}$ symmetry of the elliptic genus of K3 has attracted some attention. This development started with the observation of Eguchi et al. [1] who noted that the first few multiplicities with which the $\mathcal{N}=4$ characters appear in the elliptic genus of K3 are sums (with integer coefficients) of dimensions of representations of the Mathieu group $\mathbb{M}_{24}$. The appearance of these dimensions suggests that the underlying vector space (consisting of the states that contribute to the elliptic genus) carries an action of $\mathbb{M}_{24}$. Assuming this group action one can then also define the "twining genera" ${ }^{1}$, i.e., the elliptic genus with the insertion of a group element $g \in \mathbb{M}_{24}$,

$$
\operatorname{Tr}_{\mathrm{RR}}\left(g y^{J_{0}} q^{L_{0}-\frac{c}{24}}(-1)^{F} \bar{q}^{\bar{L}_{0}-\frac{c}{24}}(-1)^{\bar{F}}\right),
$$

and the usual string arguments suggest that these twining genera must have good modular properties under some congruence subgroup of $S L(2, \mathbb{Z})$. Using these modular properties as well as the explicit knowledge of the first few coefficients that follow from the observation of [1], all twining genera could be determined [3-6]. In turn, this leads to a stringent test of the proposal: knowing all twining genera one can deduce the decomposition of all multiplicity spaces into $\mathbb{M}_{24}$ representations, and it was found that at least for the first 1000 coefficients, non-negative integer multiplicities appear $[5,6] .{ }^{2}$ This analysis therefore gives very convincing evidence for a hidden $\mathbb{M}_{24}$ symmetry underlying the elliptic genus of K3. Further support for this conjecture was given in [7] (see also [8,9]).

\footnotetext{
${ }^{1}$ These are the analogues of the so-called MacKay-Thompson series for Monstrous Moonshine, see [2] for a modern review.

${ }^{2}$ We thank Yuji Tachikawa for informing us that he has now checked the decomposition for the first 1000 coefficients.
} 
Part of this symmetry can be understood geometrically. First of all, the elliptic genus is independent of the specific point in the moduli space of K3 that is considered, and thus the symmetries of the elliptic genus are in some sense the union of all symmetries that are present at different points in moduli space. The geometrical symmetries of K3, i.e., the symplectic automorphisms, have been studied some time ago by Mukai and Kondo $[10,11]$, and they found that at any point in moduli space these symmetries form a subgroup of $\mathbb{M}_{23}$. The Mathieu group $\mathbb{M}_{23}$ is a maximal subgroup of $\mathbb{M}_{24}$, and thus this argument "explains" part of the observation of [1].

As is familiar for example from T-duality, string theory typically has more than just the geometric symmetries, and one may therefore expect that the remaining symmetries of $\mathbb{M}_{24}$ may be accounted for by "stringy symmetries" (see also [12]). In order to analyse this question, we study in this paper the stringy version of the Mukai-Kondo theorem. More specifically, we classify the spacetime supersymmetry preserving automorphisms of the non-linear $\sigma$-model at an arbitrary point in the moduli space of K3. From the point of view of the worldsheet, these symmetries are characterized by the property that they preserve the $\mathcal{N}=(4,4)$ superconformal algebra, as well as the spectral flow operators in the RR sector. Given the observation of [1], one may have expected that all these symmetries should form a subgroup of $\mathbb{M}_{24}$, but actually the answer is more complicated:

Theorem 1.1. Let $G$ be the group of symmetries of a non-linear $\sigma$-model on $K 3$ preserving the $\mathcal{N}=(4,4)$ superconformal algebra as well as the spectral flow operators in the $R R$ sector. Then one of the following possibilities holds:

(i) $G=G^{\prime} \cdot G^{\prime \prime}$, where $G^{\prime}$ is a subgroup of $\mathbb{Z}_{2}^{11}$, and $G^{\prime \prime}$ is a subgroup of $\mathbb{M}_{24}$ with at least four orbits when acting as a permutation on $\{1, \ldots, 24\}$

(ii) $G=5^{1+2} \cdot \mathbb{Z}_{4}$

(iii) $G=\mathbb{Z}_{3}^{4} \cdot A_{6}$

(iv) $G=3^{1+4} \cdot \mathbb{Z}_{2} \cdot G^{\prime \prime}$, where $G^{\prime \prime}$ is either trivial, $\mathbb{Z}_{2}, \mathbb{Z}_{2}^{2}$ or $\mathbb{Z}_{4}$.

Here $p^{1+2 n}$ denotes an extra special group of order $p^{1+2 n}$, and N.Q denotes a group $G$ for which $N$ is a normal subgroup such that $G / N \cong Q$ (for an exposition of our mathematical notation and conventions see Appendix A). Note that except for case (i) with $G^{\prime}$ trivial, these groups are not subgroups of $\mathbb{M}_{24}$; in particular, for cases (ii)-(iv) this follows from the fact that their order does not divide

$$
\left|\mathbb{M}_{24}\right|=2^{10} \cdot 3^{3} \cdot 5 \cdot 7 \cdot 11 \cdot 23 .
$$


On the other hand, all groups in (i)-(iv) are subgroups of the Conway group $\mathrm{Co}_{1}$, and thus the analogue of the Mukai theorem is that the stringy symmetries all lie in $\mathrm{Co}_{1}$. One may take this as evidence that the elliptic genus of $\mathrm{K} 3$ should in fact have a hidden $\mathrm{Co}_{1}$ symmetry, but from its decomposition in terms of $\mathcal{N}=4$ elliptic genera, we have not seen any hint for this. In any case, the result of the Theorem means that the explanation of the $\mathbb{M}_{24}$ symmetry appearing in the elliptic genus of K3 must be more subtle.

\subsection{Sketch of proof}

Let us briefly sketch the proof of the Theorem, before returning to more general considerations below; the details of this argument as well as the underlying assumptions will be spelled out in Section 2 and Appendix B.

The basic strategy of the proof follows closely the proof of the Mukaitheorem, given by Kondo. The moduli space of sigma-models on K3 has the form

$$
\mathcal{M}_{\mathrm{K} 3}=O\left(\Gamma^{4,20}\right) \backslash O(4,20) /(O(4) \times O(20)) .
$$

Here the Grassmannian $O(4,20) /(O(4) \times O(20))$ parameterizes the choice of a positive definite four-dimensional subspace $\Pi \subset \mathbb{R}^{4,20}$, and $O\left(\Gamma^{4,20}\right)$ is the group of automorphisms of the even unimodular lattice $\Gamma^{4,20} \subset \mathbb{R}^{4,20}$ of signature $(4,20)$. We may think of $\Gamma^{4,20}$ as the integral homology of K3, i.e., as the D-brane charge lattice, while the position of $\Pi$ is specified by the choice of a Ricci-flat metric and a $B$-field on K3. In particular, it therefore determines the four left- and right-moving supercharges.

The supersymmetry preserving automorphisms of the non-linear $\sigma$-model characterized by $\Pi$ generate the group $G \equiv G_{\Pi}$ that consists of those elements of $O\left(\Gamma^{4,20}\right)$ that leave $\Pi$ pointwise fixed. We denote by $L^{G}$ the sublattice of $G$-invariant vectors of $L \equiv \Gamma^{4,20}$, and define $L_{G}$ to be its orthogonal complement. By construction, $\Pi$ is a subspace of the real vector space $L^{G} \otimes \mathbb{R} \subset \mathbb{R}^{4,20}$, and since $\Pi$ has signature $(4,0)$, the orthogonal complement $L_{G}$ must be a negative definite lattice of rank at most 20 . The basic idea is now to embed $L_{G}(-1)$ - the $(-1)$ means that we change the sign of its intersection matrix - into the Leech lattice $\Lambda$. Such an embedding exists, provided we assume that $L_{G}(-1)$ does not contain any vectors of length squared two (which would signal some gauge enhancement and thus would lead to a singular CFT). Since the action of $G$ fixes all vectors of $\Lambda$ orthogonal to $L_{G}(-1)$, it follows that $G$ must be a subgroup of $\mathrm{Co}_{0}=\operatorname{Aut}(\Lambda)$ that fixes pointwise a sublattice of the Leech lattice of rank at least 4 . 
A more careful analysis then shows that $G$ is isomorphic to a subgroup of $\mathrm{Co}_{1} \cong \mathrm{Co}_{0} / \mathbb{Z}_{2}$ and leads to the separate cases (i)-(iv) above.

\subsection{Comments and outline}

Since the result of the Theorem is somewhat contrary to expectations, we have also studied a few explicit conformal field theories describing K3 at different points in moduli space in detail. In particular, we have done this for (A) the orbifold point $\mathbb{T}^{4} / \mathbb{Z}_{2} ;$ (B) the orbifold point $\mathbb{T}^{4} / \mathbb{Z}_{4}$ which is equivalent to the Gepner model $(2)^{4}$; and (C) the Gepner model $(1)^{6}$. Given that these descriptions are very explicit, it is possible to identify (at least some of) the supersymmetry-preserving automorphisms. In each case we have computed the resulting symmetry group, and compared it with the possibilities allowed for by the Theorem. We find that (C) realizes case (iii), while both (A) and (B) correspond to case (i) with $G^{\prime}$ non-trivial. In particular, all of these cases therefore describe K3s for which the stringy symmetries do not lie inside $\mathbb{M}_{24}$.

From the point of view of the argument leading to the Theorem, the symmetries of the worldsheet theory should have an interpretation as lattice symmetries. Actually, this point of view can also be directly understood in conformal field theory: as mentioned before, the lattice $\Gamma^{4,20}$ can be identified with the D-brane charge lattice, and $\Pi$ describes the four left- and rightmoving supercharges. Thus the supersymmetry preserving automorphisms of the CFT should be in one-to-one correspondence with the symmetries of the D-brane charge lattice that leave $\Pi$ pointwise invariant. Using conformal field theory methods, it is fairly straightforward to determine the D-brane charge lattice, as well as the $\Pi$-preserving symmetries. For each of the three cases $(\mathrm{A})-(\mathrm{C})$ we have verified that the resulting symmetry groups reproduce precisely those obtained from the explicit construction of the symmetry generators above. Incidentally, this method to determine the supersymmetry preserving automorphisms by analysing the D-brane charge lattice constitutes a nice general approach that can be applied to any non-linear $\sigma$-model on K3.

The paper is organized as follows. In the following section (Section 2), we give a more detailed description of the main Theorem and the assumptions that go into its proof. Section 3 is devoted to the study of the orbifold point $\mathbb{T}^{4} / \mathbb{Z}_{2}$. Among other things, we calculate the twining genera for the various symmetries and find the twining genus of the $2 \mathrm{~B}$ conjugacy class of $\mathbb{M}_{24}$ that does not lie inside $\mathbb{M}_{23}$. (The corresponding symmetry is the stringy fourfold T-duality symmetry $\mathcal{T}$, see (3.14).) However, we also find symmetries 
that do not lie inside $\mathbb{M}_{24}$, and whose twining genus does not agree with the twining genus of any conjugacy class in $\mathbb{M}_{24}$. (The simplest example is the "quantum symmetry" $\mathcal{Q}$, see (3.18).) In Section 4, the same analysis is done for the $(1)^{6}$ Gepner point, while Section 5 deals with the $(2)^{4}$ Gepner model. Our notation and some basic mathematical background is described in Appendix A, while some of the details of the proof of the Theorem have been delegated to Appendix B. Finally, Appendix C contains some of the details of the D-brane charge analysis for the Gepner models.

\section{Symmetries of non-linear $\sigma$-models on K3}

In the following, we shall consider two-dimensional theories with $\mathcal{N}=(4,4)$ superconformal symmetry and central charge $c=6$. Theories of this type can be classified according to their elliptic genus $\phi(\tau, z)$. The symmetries of the theory constrain the elliptic genus to be a weak Jacobi form of weight 0 and index 1 . The only possibilities are $\phi(\tau, z)=0$, which corresponds to the case of the target space being $\mathbb{T}^{4}$, or

$$
\phi(\tau, z)=8 \sum_{i=2}^{4} \frac{\vartheta_{i}(\tau, z)^{2}}{\vartheta_{i}(\tau, 0)^{2}}=2 y+20+2 y^{-1}+O(q)
$$

where $\vartheta_{i}$ are the Jacobi theta functions and the normalization is fixed by the condition that the vacuum is unique. This second case arises if the target space is K3 and it is the main focus of the present paper.

\subsection{Moduli space of non-linear $\sigma$-models on K3}

As mentioned in the introduction, the moduli space of $\mathcal{N}=(4,4)$ theories with elliptic genus (2.1) is believed to be the quotient (see for example $[13,14])$

$$
\mathcal{M}_{\mathrm{K} 3}=O\left(\Gamma^{4,20}\right) \backslash O(4,20) /(O(4) \times O(20)) .
$$

Here the Grassmannian $O(4,20) /(O(4) \times O(20))$ parameterizes the choice of a positive definite four-dimensional subspace in $\mathbb{R}^{4,20}$, and $O\left(\Gamma^{4,20}\right)$ is the group of automorphisms of the even unimodular lattice $\Gamma^{4,20}$ with signature $(4,20) .^{3}$

\footnotetext{
${ }^{3}$ This description is based on the hypothesis that all $\mathcal{N}=(4,4)$ superconformal field theories with elliptic genus (2.1) can be realized as non-linear $\sigma$-models on a
} 
Geometrically, we can think of $\Gamma^{4,20}$ as the integral homology lattice $H_{\text {even }}(X, \mathbb{Z})$ of the $\mathrm{K} 3$ manifold $X$, with the bilinear form given by the intersection number. The space $\mathbb{R}^{4,20}$ is interpreted as the real even cohomology $H^{\text {even }}(X, \mathbb{R})$ endowed with the cup product, and the embedding $\Gamma^{4,20} \subset$ $\mathbb{R}^{4,20}$ is realized through Poincaré duality $H_{\text {even }}(X, \mathbb{Z}) \cong H^{\text {even }}(X, \mathbb{Z}) \subset$ $H^{\text {even }}(X, \mathbb{R})$. The non-linear $\sigma$-model is determined by choosing a Ricci-flat metric and a B-field on the manifold $X$; this corresponds to the choice of the four-dimensional subspace $\Pi \subset H^{\text {even }}(X, \mathbb{R})$, although the relationship is rather involved (see e.g. [13]).

In string theory, the homology lattice can be identified with the lattice of D-brane charges, and the intersection number for $\alpha, \beta \in H_{\text {even }}(X, \mathbb{Z})$ is reproduced by the overlap

$$
\alpha \cdot \beta=\left\langle\left\langle\alpha\left\|q^{\frac{1}{2}\left(L_{0}+\tilde{L}_{0}\right)-\frac{c}{12}}(-1)^{F_{L}}\right\| \beta\right\rangle_{\mathrm{RR}} .\right.
$$

Here only the $\mathrm{RR}$ part of the boundary states $\| \alpha\rangle\rangle, \| \beta\rangle$ corresponding to the D-branes wrapping the cycles $\alpha$ and $\beta$ contribute [15]. (Alternatively, (2.3) is the Witten index in the $\mathrm{R}$-sector of the relative open string.) In this picture, the dual space of real cohomology is naturally identified with the space of 24 (anti-)chiral RR ground states with $h=\bar{h}=\frac{1}{4}$. Under the action of $\mathrm{SU}(2)_{\mathrm{L}} \times \mathrm{SU}(2)_{\mathrm{R}}$, which is part of the $\mathcal{N}=(4,4)$ superconformal symmetry, the $24 \mathrm{RR}$ ground states split into a four-dimensional $(\mathbf{2}, \mathbf{2})$ representation and 20 singlets. The four-dimensional subspace $\Pi \subset \mathbb{R}^{4,20}$ is then to be identified with the subspace of RR states transforming in the $(\mathbf{2}, \mathbf{2})$-representation.

\subsection{Characterization of $\mathcal{N}=(4,4)$ preserving symmetries}

Our goal is to classify the discrete symmetries $\tilde{G}_{\Pi}$ of a given $\mathcal{N}=(4,4)$ theory parameterized by $\Pi$ in the moduli space $\mathcal{M}_{\mathrm{K} 3}$. Any symmetry $g \in \tilde{G}_{\Pi}$ must obviously leave $\Pi$ invariant. It must therefore either be an element of $O\left(\Gamma^{4,20}\right)$, or it must act trivially on the Grassmannian in (2.2). However, the latter case would imply that the symmetry exists everywhere in moduli space, and we know (for example from studying deformations of the orbifold line) that this is not possible. Thus we conclude that the symmetries $\tilde{G}_{\Pi}$ of the theory at $\Pi$ is precisely the subgroup of $O\left(\Gamma^{4,20}\right) \subset O(4,20, \mathbb{R})$ that leaves $\Pi$ (setwise) fixed.

K3 manifold. Although this conjecture is widely accepted in the literature, it has not yet been proven (see [14]). We will implicitly assume that the conjecture holds in the rest of the paper. 
A general symmetry $g \in \tilde{G}_{\Pi}$ will preserve the $\mathcal{N}=(4,4)$ superconformal algebra only up to an automorphism. From now on we want to focus on the subgroup $G_{\Pi} \subset \tilde{G}_{\Pi}$ that actually leaves the $\mathcal{N}=(4,4)$ superconformal algebra invariant, i.e., for which this automorphism is trivial. This is motivated by the requirement that the spectral flow operators are responsible for spacetime supersymmetry [16] and hence should be left invariant; at $c=6$ they correspond precisely to the currents extending the $u(1)$ subalgebra of the $\mathcal{N}=2$ algebra to the $s u(2)$ algebra of the $\mathcal{N}=4$ algebra. In addition, we demand that the RR-states that correspond to these $s u(2)$ currents under the spectral flow are indeed invariant. ${ }^{4}$ These symmetries are then relevant for the analysis of "Mathieu Moonshine" [1] that was reviewed at the beginning of the Introduction. Indeed, $\mathbb{M}_{24}$ appears to act on the multiplicity spaces with which the $\mathcal{N}=4$ representations appear in the elliptic genus, and hence must commute with the left-moving $\mathcal{N}=4$ superconformal algebra. Furthermore, according to the proposal of [3,4], the four RR ground states that transform in the $(\mathbf{2}, \mathbf{2})$ of the $\mathrm{SU}(2)_{\mathrm{L}} \times \mathrm{SU}(2)_{\mathrm{R}}$ - these are related to the spacetime supercharges - sit in a singlet representation of $\mathbb{M}_{24}$. Thus the symmetries that are described by $\mathbb{M}_{24}$ should leave the full $\mathcal{N}=(4,4)$ superconformal algebra invariant, and preserve the spectral flow operators in the RR sector. Since $\mathbb{M}_{24}$ is not a subgroup of $O\left(\Gamma^{4,20}\right)^{5}$, we cannot explain the full $\mathbb{M}_{24}$ symmetry by looking at just one point in moduli space. However, the elliptic genus is constant over moduli space, and one may therefore expect that we can account for the entire $\mathbb{M}_{24}$ by putting information from different points in moduli space together. This is one of the main motivations for classifying the symmetry groups at different points in moduli space.

In any case, since $\Pi \subset \mathbb{R}^{4,20}$ can be identified with the subspace of $R R$ states transforming in the $(\mathbf{2}, \mathbf{2})$-representation, we conclude that

The subgroup $G_{\Pi}$ of symmetries of the $K 3$ o-model charac-
terized by $\Pi \subset \mathbb{R}^{4,20}$ that leave the $\mathcal{N}=(4,4)$ superconformal

\footnotetext{
${ }^{4}$ This is not automatic since we may modify our symmetry by the inclusion of $(-1)^{F_{s}}$ which only changes the sign of the left-moving R-sector.

${ }^{5}$ This can be proven as follows. The only non-trivial action of $\mathbb{M}_{24}$ on $\mathbb{R}^{24}$ is as a five-transitive permutation group on the vectors $v_{1}, \ldots, v_{24}$ of a suitable basis. Suppose that this action preserves a symmetric bilinear form $(\cdot, \cdot)$ on $\mathbb{R}^{24}$ and set $A_{i j}:=\left(v_{i}, v_{j}\right)$. Five-transitivity implies that the entries $A_{i j}$ form two orbits under the induced action of $\mathbb{M}_{24}$, the diagonal and the off-diagonal ones. If we set $x:=A_{i i}$ and $y:=A_{i j}, i \neq j$, then $x-y$ is an eigenvalue of $A$ with multiplicity at least 23 , so that $A$ cannot have signature $(4,20)$.
} 
algebra invariant and that preserve the spectral flow operators in the $R R$ sector, is the subgroup of $O\left(\Gamma^{4,20}\right) \subset O(4,20, \mathbb{R})$ that leaves $\Pi$ (pointwise) fixed.

In the following, by a symmetry of an $\mathcal{N}=(4,4)$ theory in $\mathcal{M}_{\mathrm{K} 3}$, we will always mean a transformation with these properties. Note that this restriction excludes some very interesting symmetries, for example, mirror symmetry in a self-mirror theory. On the other hand, the extension of our arguments to more general cases is fairly straightforward.

\subsection{Classification of the groups of symmetries}

In this section, we will classify the possible groups $G_{\Pi}$. The related problem in classical geometry has been previously considered by Mukai [10], who classified the groups of symplectic automorphisms of K3 surfaces. Mukai proved that the symplectic automorphisms of any K3 surface form a subgroup of $\mathbb{M}_{23}$, which in turn is a maximal subgroup of $\mathbb{M}_{24}$. The Mathieu group $\mathbb{M}_{23}$ is finite and its subgroups are well studied, so that the Mukai theorem provides a very explicit description of all symplectic automorphisms. In the following, we will extend the Mukai theorem to the classification of the symmetry groups of the $\sigma$-models. In particular, we will show that $G_{\Pi} \subset \mathrm{Co}_{1}$, the Conway group $\mathrm{Co}_{1}$. Note that $\mathrm{Co}_{1}$ contains $\mathbb{M}_{23}$ (as well as $\mathbb{M}_{24}$ ) as a subgroup.

As argued in the previous section, the symmetries of interest form the subgroup of $O\left(\Gamma^{4,20}\right) \subset O(4,20, \mathbb{R})$ that fix (pointwise) the positive-definite four-dimensional subspace $\Pi \subset \mathbb{R}^{4,20}$, characterizing the relevant point in moduli space. However, not all choices of $\Pi$ correspond to well-defined conformal field theories. In particular, when $\Pi$ is orthogonal to a vector $v \in \Gamma^{4,20}$ of norm $v^{2}=-2$ (usually called a root of $\Gamma^{4,20}$ ), the corresponding nonlinear $\sigma$-model is not well defined [17]. This subtlety can be understood by considering the model as an internal CFT in type IIA superstring theory. This theory is dual to heterotic string theory compactified on $\mathbb{T}^{4}$. Generically, the corresponding low-energy effective field theory contains an abelian gauge group $U(1)^{24}$. However, when $\Pi$ is orthogonal to a root $v$, the gauge group is enhanced to a non-abelian gauge group, and $v$ is interpreted as a root of the corresponding Lie algebra. The additional states in type IIA superstring theory are interpreted as D-branes becoming massless at this point of the moduli space. This means that the corresponding perturbative superconformal field theory cannot describe correctly all massless degrees of freedom of the theory, and hence the non-linear $\sigma$-model is expected to be 
inconsistent [18]. Therefore, in the following, we shall exclude the points in the moduli space where $\Pi$ is orthogonal to a root. It is believed that these are the only singular points in the moduli space.

Our strategy to characterize the groups $G_{\Pi}$ is inspired by the proof of the Mukai theorem given by Kondo [11]. Let $\Pi \subset \mathbb{R}^{4,20}$ be a four-dimensional positive definite space, not orthogonal to any root (vector of norm -2) in $L \equiv \Gamma^{4,20} \subset \mathbb{R}^{4,20}$, and let $G \equiv G_{\Pi} \subset O\left(\Gamma^{4,20}\right)$ be the subgroup of lattice automorphisms fixing $\Pi$ pointwise. We denote by $L^{G}$ the sublattice of vectors fixed by $G$

$$
\Gamma^{4,20} \supset L^{G}:=\left\{v \in \Gamma^{4,20} \mid g(v)=v, \quad \text { for all } g \in G\right\},
$$

and by $L_{G}$ its orthogonal complement

$$
\Gamma^{4,20} \supset L_{G}:=\left\{w \in \Gamma^{4,20} \mid w \cdot v=0, \quad \text { for all } v \in L^{G}\right\} .
$$

By definition, the real vector space $L^{G} \otimes \mathbb{R} \subset \mathbb{R}^{4,20}$ will contain $\Pi, \Pi \subset$ $L^{G} \otimes \mathbb{R}$, and since $\Pi$ has signature $(4,0)$, the orthogonal complement $L_{G}$ must be negative definite and have rank at most 20. Furthermore, every vector in $L_{G}$ is orthogonal to $\Pi$, so that, by our assumption on $\Pi, L_{G}$ contains no roots. The proof of the Theorem then proceeds as follows - the relevant details are given in Appendix B:

- First (see Appendix B.1), we prove that $L_{G}(-1)$, can be embedded in the even unimodular lattice $\Gamma^{25,1}$. The action of $G$ on $L_{G}$ extends to an action on $\Gamma^{25,1}$, which fixes all vectors orthogonal to $L_{G}(-1)$ in $\Gamma^{25,1}$. Thus, $G$ is a subgroup of $\operatorname{Aut}\left(\Gamma^{25,1}\right)$.

- Next (see Appendix B.2), using the fact that $L_{G}(-1)$ contains no vectors of norm 2 and the properties of $\operatorname{Aut}\left(\Gamma^{25,1}\right)$, we show that $L_{G}(-1)$ must be contained in a positive definite sublattice of $\Gamma^{25,1}$, namely the Leech lattice $\Lambda$. This is the unique 24-dimensional even unimodular lattice with no roots and its group of automorphisms is the Conway group $\mathrm{Co}_{0}$ [19]. This group can be obtained by extending the sporadic finite simple group $\mathrm{Co}_{1}$ [20] of order

$$
\left|\mathrm{Co}_{1}\right|=2^{21} \cdot 3^{9} \cdot 5^{4} \cdot 7^{2} \cdot 11 \cdot 13 \cdot 23 \sim 4 \times 10^{18},
$$

by the $\mathbb{Z}_{2}$ symmetry that changes the sign of all vectors in $\Lambda$ (a central element in $\left.\mathrm{Co}_{0}\right)$. Once again, the action of $G$ on $L_{G}(-1)$ can be extended to an action on $\Lambda$ which fixes all vectors orthogonal to $L_{G}(-1)$. This means, in particular, that the $\mathbb{Z}_{2}$ symmetry that changes 
the sign of all vectors in $\Lambda$ is not an element of $G$, since it has no nontrivial fixed vectors. Thus, $G$ is a subgroup of $\mathrm{Co}_{0}$ and the natural homomorphism $\mathrm{Co}_{0} \rightarrow \mathrm{Co}_{0} / \mathbb{Z}_{2} \cong \mathrm{Co}_{1}$ induces an injective homomorphism of $G$ into $\mathrm{Co}_{1}$. It follows that $G$ is isomorphic to a subgroup of the finite simple group $\mathrm{Co}_{1}$.

Combining these results thus leads to the natural analogue of the Mukai theorem:

Proposition 2.1. The group $G_{\Pi}$ of symmetries of any non-linear $\sigma$-model on $K 3$ is a subgroup of the Conway group $\mathrm{Co}_{1} \cong \operatorname{Aut}(\Lambda) / \mathbb{Z}_{2}$ that fixes pointwise a sublattice of the Leech lattice $\Lambda$ of rank at least 4.

In order to give a more precise description of the groups of symmetries $G_{\Pi}$, one needs a detailed classification of the subgroups of $\mathrm{Co}_{0}$ that fix a sublattice of rank 4 in $\Lambda$. The result of this somewhat technical analysis the details are explained in Appendix B.3 - is the Theorem stated in the Introduction.

We should mention that our analysis does not actually prove that $\mathrm{Co}_{1}$ is the smallest possible group containing all these symmetry groups. However, some simple considerations on the order of the groups $\left|G_{\Pi}\right|$ are sufficient to exclude all maximal subgroups of $\mathrm{Co}_{1}$, except for $\mathrm{Co}_{2}$. Furthermore, all the cases (i)-(iv) in the Theorem are actually realized by some $\mathcal{N}=(4,4)$ model, provided we assume that every four-dimensional subspace $\Pi \subset \mathbb{R}^{4,20}$, not orthogonal to any vector of norm -2 in $\Gamma^{4,20}$, leads to a consistent conformal field theory (see Appendix B.4). The characterization of the symmetry groups as given in the Theorem is therefore optimal.

In the following, we shall describe in detail specific examples that realize some of the possibilities of the Theorem. In particular, the $\mathbb{T}^{4} / \mathbb{Z}_{2}$ orbifold model to be discussed in Section 3 and the $(2)^{4}$ Gepner model of Section 5 are examples of case (i) and have symmetry groups that are not subgroups of $\mathbb{M}_{24}$, while the Gepner model $(1)^{6}$ that will be studied in Section 4 realizes precisely case (iii).

\section{The $\mathbb{Z}_{2}$ orbifold model}

In order to illustrate the general predictions of the Theorem let us consider a few specific examples. We begin with the $\mathbb{T}^{4} / \mathbb{Z}_{2}$ orbifold model where we take $\mathbb{T}^{4}$ to be the orthogonal torus at the self-dual radius, i.e., the four radii take on the self-dual value, and set the $B$-field on the torus to zero. We write $\mathbb{T}^{4}=\mathbb{T}^{2} \times \mathbb{T}^{2}$, and label the two $\mathbb{T}^{2} \mathrm{~s}$ by $i=1,2$. For each $\mathbb{T}^{2}$ we use complex 
coordinates, and thus the left-moving bosonic and fermionic modes are

$$
\alpha_{n}^{(i)}, \quad \bar{\alpha}_{n}^{(i)}, \quad \psi_{n}^{(i)}, \quad \bar{\psi}_{n}^{(i)}, \quad i=1,2,
$$

and similarly for the right-movers. (The right-movers are denoted by a tilde.) The $\mathbb{Z}_{2}$ orbifold $\mathcal{I}$ acts as -1 an all of these modes. In addition, it maps the momentum ground states $\mathcal{I}\left(p_{\mathrm{L}}, p_{\mathrm{R}}\right)=\left(-p_{\mathrm{L}},-p_{\mathrm{R}}\right)$.

\subsection{The spectrum in the $R R$ sector}

We denote the states in the untwisted sector of $\mathbb{T}^{4}=\mathbb{T}^{2} \times \mathbb{T}^{2}$ by

$$
\left|p_{\mathrm{L}}, p_{\mathrm{R}} ; N, \tilde{N} ; s ; \tilde{s}\right\rangle
$$

where $p_{\mathrm{L}}=(n+w) / \sqrt{2}, p_{\mathrm{R}}=(n-w) / \sqrt{2}$ are the left and right momenta at the self dual radius, with $n \in \mathbb{Z}^{4}$ the momentum and $w \in \mathbb{Z}^{4}$ the winding numbers. Furthermore, $N$ and $\tilde{N}$ denote the left and right oscillator contributions, while $s, \tilde{s}$ label the Ramond ground states. More specifically,

$$
(s ; \tilde{s})=\left(s_{1}, s_{2} ; \tilde{s}_{1}, \tilde{s}_{2}\right),
$$

where each $s_{i}, i=1,2$, can take the two values $\pm \frac{1}{2}$, and the zero modes $\bar{\psi}_{0}^{(i)}$ and $\psi_{0}^{(i)}$ map the states with $s_{i}= \pm \frac{1}{2}$ into one another; the analogous statement holds for the $\tilde{s}_{i}$ in the right-moving sector. The RR ground states have charge $(S, \tilde{S})$ with respect to the left- and right-moving $U(1)$-current, where

$$
S=\sum_{i} s_{i}, \quad \tilde{S}=\sum_{i} \tilde{s}_{i} .
$$

To obtain the spectrum of the $\mathbb{Z}_{2}$-orbifold theory we have to project onto states that are even under the operator $\mathcal{I}$ acting as

$$
\mathcal{I}\left|p_{\mathrm{L}}, p_{\mathrm{R}} ; N, \tilde{N} ; s ; \tilde{s}\right\rangle=(-1)^{|N|+|\tilde{N}|}(-1)^{S+\tilde{S}}\left|-p_{\mathrm{L}},-p_{\mathrm{R}} ; N, \tilde{N} ; s ; \tilde{s}\right\rangle
$$

where $|N|$ is the total number of oscillators appearing in $N$, and likewise for $|\tilde{N}|$.

In the twisted sector the states are labelled by

$$
|i ; N, \tilde{N}\rangle
$$

where $i=1, \ldots, 16$ distinguishes the 16 different fixed points of the orbifold, while $N, \tilde{N}$ denote again the oscillator numbers. The ground states do not 
carry any charge with respect to the left- and right-moving $U(1)$-currents. The orbifold projection in the twisted sector acts as

$$
\mathcal{I}|i ; N, \tilde{N}\rangle=(-1)^{|N|+|\tilde{N}|}|i ; N, \tilde{N}\rangle .
$$

It is straightforward to calculate the elliptic genus from this description [21]. In the untwisted sector one finds

$$
\phi^{(U)}(\tau, z)=\left(2 y+4+2 y^{-1}\right) \prod_{n=1}^{\infty} \frac{\left(1+q^{n} y\right)^{2}\left(1+q^{n} y^{-1}\right)^{2}}{\left(1+q^{n}\right)^{4}}=8 \frac{\vartheta_{2}(\tau, z)^{2}}{\vartheta_{2}(\tau, 0)^{2}},
$$

while the contribution of the twisted sector equals

$$
\phi^{(T)}(\tau, z)=8\left(\frac{\vartheta_{4}(\tau, z)^{2}}{\vartheta_{4}(\tau, 0)^{2}}+\frac{\vartheta_{3}(\tau, z)^{2}}{\vartheta_{3}(\tau, 0)^{2}}\right) .
$$

It is easy to see that their sum, $\phi^{(U)}(\tau, z)+\phi^{(T)}(\tau, z)$, reproduces precisely (2.1).

\subsection{Symmetries and twining genera}

The orbifold theory possesses various symmetries that leave the $\mathcal{N}=(4,4)$ superconformal algebra invariant and that preserve the spectral flow operators in the RR sector; in particular, we have (see [14] and references therein)

(1) $\mathcal{R}$ : rotation of the two $\mathbb{T}^{2}$ 's by $90^{\circ}$ and $-90^{\circ}$, respectively.

(2) $\mathcal{E}$ : exchanging the two $\mathbb{T}^{2}$ 's, together with an inversion acting on the second $\mathbb{T}^{2}$, say.

(3) $H_{a}$ : half-period translations, that act as $(-1)^{p \cdot a}, a \in(\mathbb{Z} / 2 \mathbb{Z})^{4}$, in the untwisted sector, and by a permutation on the 16 twisted sectors.

(4) $\mathcal{T}$ : four-fold T-duality. (Note that the two-fold T-duality induces a non-trivial automorphism of the $\mathcal{N}=(4,4)$ superconformal algebra.)

(5) $\mathcal{Q}$ : the quantum symmetry that acts as +1 on the untwisted, and as -1 on the twisted sector.

These symmetries generate the group $G=2^{1+8} \rtimes \mathbb{Z}_{2}^{3}$. The normal subgroup acting trivially on the untwisted sector RR ground states with $p_{\mathrm{L}}=p_{\mathrm{R}}=0$ is the extra special group $2^{1+8}$ of order $2^{9}$, containing $\mathcal{Q}$, the centre of $G$. It is generated by the half-shifts $H_{a}$ that form an abelian $\mathbb{Z}_{2}^{4}$, and by their 
dual symmetries $G_{a}=\mathcal{T} H_{a} \mathcal{T}$ that change the sign of half of the twisted sectors. The only non-trivial commutators are $H_{a} G_{b} H_{a} G_{b}=\mathcal{Q}^{a \cdot b}$, where $a, b \in(\mathbb{Z} / 2 \mathbb{Z})^{4}$. The quotient group $G / 2^{1+8} \cong \mathbb{Z}_{2}^{3}$ is generated by $\mathcal{R}, \mathcal{E}$ and $\mathcal{T}$. Conjugation by $\mathcal{R}$ and $\mathcal{E}$ yields a permutation of the half shifts (and the analogous permutations for $G_{a}$ ), while conjugation by $\mathcal{T}$ exchanges $G_{a}$ and $H_{a}$. The group $G=2^{1+8} \rtimes \mathbb{Z}_{2}^{3}$ realizes case (i) of the Theorem with $G^{\prime}=\mathbb{Z}_{2}^{6}$ and $G^{\prime \prime}=\mathbb{Z}_{2}^{4} \rtimes \mathbb{Z}_{2}^{2}$. Here $G^{\prime \prime} \subset \mathbb{M}_{24}$ is generated by $H_{a}, \mathcal{E}$ and $\mathcal{R}$, while $G^{\prime} \subset \mathbb{Z}_{2}^{11}$ is generated by $\mathcal{Q}$ (one $\mathbb{Z}_{2}$ factor), by the composition $\mathcal{T} \mathcal{R} \mathcal{E}$ (another $\mathbb{Z}_{2}$ ), and by the elements of the form $H_{a} \mathcal{T} \mathcal{R E} H_{a}$ (giving $\mathbb{Z}_{2}^{4}$ ).

Given the explicit description of the RR sector from above, it is straightforward to calculate the corresponding twining genera,

$$
\phi_{g}(\tau, z)=\operatorname{Tr}_{\mathrm{RR}}\left(g q^{L_{0}-\frac{1}{4}} \bar{q}^{\bar{L}_{0}-\frac{1}{4}} y^{J_{0}}(-1)^{F+\bar{F}}\right),
$$

i.e., the elliptic genus with the insertion of the symmetry $g .{ }^{6}$ In particular, we can check how these twining genera compare with the Mathieu twining genera that were worked out in [3-6]. Our explicit results are, for example,

$$
\begin{aligned}
\phi_{\mathcal{R}}(\tau, z) & =4 \frac{\vartheta_{2}(2 \tau, 2 z)}{\vartheta_{2}(2 \tau, 0)}+4 \frac{\vartheta_{3}(2 \tau, 2 z)}{\vartheta_{3}(2 \tau, 0)}=\phi_{2 \mathrm{~A}}(\tau, z) \\
\phi_{\mathcal{E}}(\tau, z) & =4 \frac{\vartheta_{2}(2 \tau, 2 z)}{\vartheta_{2}(2 \tau, 0)}+4 \frac{\vartheta_{3}(2 \tau, 2 z)}{\vartheta_{3}(2 \tau, 0)}=\phi_{2 \mathrm{~A}}(\tau, z) \\
\phi_{H_{a}}(\tau, z) & =8 \frac{\vartheta_{2}(\tau, z)^{2}}{\vartheta_{2}(\tau, 0)^{2}}=\phi_{2 \mathrm{~A}}(\tau, z) \\
\phi_{\mathcal{T}}(\tau, z) & =-2 \vartheta_{4}(2 \tau)^{4} \frac{\vartheta_{1}(\tau, z)^{2}}{\eta(\tau)^{6}}=\phi_{2 \mathrm{~B}}(\tau, z) \\
\phi_{\mathcal{T} H_{a}}(\tau, z) & =\phi_{4 \mathrm{~A}}(\tau, z) \quad\left[a^{2} \text { even, say } a=(1100)\right] \\
\phi_{\mathcal{R} H_{a}}(\tau, z) & =\phi_{4 \mathrm{~B}}(\tau, z) \quad[\text { e.g., for } a=(1111)] \\
\phi_{\mathcal{T} H_{a}}(\tau, z) & =\phi_{4 \mathrm{C}}(\tau, z) \quad[\text { e.g., for } a=(1000)]
\end{aligned}
$$

\footnotetext{
${ }^{6}$ The condition that the symmetry preserves the full $\mathcal{N}=(4,4)$ superconformal algebra guarantees that the resulting twining genus still defines a weak Jacobi form. Symmetries that only preserve the $\mathcal{N}=(2,2)$ superconformal algebra typically act non-trivially on $J^{ \pm}$, and then the resulting twining genera do not have the shift symmetry under $z \mapsto z+\tau$.
} 


$$
\begin{aligned}
\phi_{\mathcal{Q}}(\tau, z) & =8\left(\frac{\vartheta_{2}(\tau, z)^{2}}{\vartheta_{2}(\tau, 0)^{2}}-\frac{\vartheta_{3}(\tau, z)^{2}}{\vartheta_{3}(\tau, 0)^{2}}-\frac{\vartheta_{4}(\tau, z)^{2}}{\vartheta_{4}(\tau, 0)^{2}}\right) \\
& =2 \phi_{2 \mathrm{~A}}(\tau, z)-\phi_{1 \mathrm{~A}}(\tau, z)
\end{aligned}
$$

$$
\begin{aligned}
\phi_{\mathcal{Q} \mathcal{T} H_{a} G_{b}}(\tau, z) & =-2 \frac{\vartheta_{3}(2 \tau)^{4} \vartheta_{1}(\tau, z)^{2}}{\eta(\tau)^{6}}-2 \frac{\vartheta_{3}(\tau, z)^{2}}{\vartheta_{3}(\tau)^{2}}-2 \frac{\vartheta_{4}(\tau, z)^{2}}{\vartheta_{4}(\tau)^{2}} \\
& =\frac{1}{2}\left(-\phi_{1 \mathrm{~A}}+\phi_{2 \mathrm{~A}}+2 \phi_{4 \mathrm{~B}}\right) \quad[\text { e.g., for } a=(1100), b=(0011)] .
\end{aligned}
$$

It is worth pointing out that the generators $H_{a}, \mathcal{E}$ and $\mathcal{R}$ that generate $G^{\prime \prime} \subset$ $\mathbb{M}_{24}$ lead to twining genera that directly agree with $\mathbb{M}_{24}$ twining genera. On the other hand, the twining genera of the quantum symmetry $\mathcal{Q}$, see (3.18), and of the group elements $g=\mathcal{Q} \mathcal{T} H_{a} G_{b}$ for suitable choices of $a$ and $b$, see (3.19), do not equal any Mathieu twining genus (but can only be expressed in terms of linear combinations of such twining genera). Finally, certain twining genera involving the four-fold T-duality, namely $\mathcal{T}$ and $\mathcal{T} H_{a}$ for suitable $a$, give rise to twining genera, whose conjugacy classes $(2 \mathrm{~B}, 4 \mathrm{~A}$ and $4 \mathrm{C})$ do not lie inside $\mathbb{M}_{23}$. This ties in with the fact that $\mathrm{T}$-duality is a non-geometric symmetry.

\subsection{The D-brane charge lattice}

As suggested by the proof of the Theorem (and explained in Section 2), we should also be able to characterize the symmetry group of the model as the symmetries of the D-brane charge lattice that leave the four-dimensional subspace $\Pi$ (corresponding to the four supercharges) invariant. For the case at hand, the D-brane charge lattice can be computed straightforwardly since the primitive branes may be taken to be fractional D0, D2 and D4 branes. They can be constructed as explained, for example, in [22] (see also [23,24]). If we denote the Ishibashi states in the untwisted and the $i$ th twisted sector by $|\mathrm{Dp}, \mathrm{U}\rangle\rangle$ and $|\mathrm{Dp}, i\rangle\rangle$, respectively, the fractional branes have the structure

$$
\begin{aligned}
\| \mathrm{D} 4, \epsilon, \delta\rangle\rangle_{\mathrm{f}} & \left.\left.=\frac{1}{2}|\mathrm{D} 4, \mathrm{U}\rangle\right\rangle+\frac{\epsilon}{4} \sum_{i=1}^{16} \delta_{i}|\mathrm{D} 4, i\rangle\right\rangle, \\
\| \mathrm{D} 2(j k), \epsilon, \delta\rangle\rangle_{\mathrm{f}} & \left.\left.=\frac{1}{2}|\mathrm{D} 2(j k), \mathrm{U}\rangle\right\rangle+\frac{\epsilon}{2} \sum_{i \in P_{j k}} \delta_{i}|\mathrm{D} 2(j k), i\rangle\right\rangle,
\end{aligned}
$$




$$
\left.\left.\| \mathrm{D} 0, i, \epsilon\rangle\rangle_{\mathrm{f}}=\frac{1}{2}|\mathrm{D} 0, \mathrm{U}\rangle\right\rangle+\epsilon|\mathrm{D} 0, i\rangle\right\rangle,
$$

where $\| \mathrm{D} 0, i, \epsilon\rangle\rangle_{\mathrm{f}}$ is the fractional D0-brane at the $i$ th fixed point, while $\| \mathrm{D} 2(j k), \epsilon, \delta\rangle\rangle_{\mathrm{f}}$ denotes the fractional D2-branes oriented along the $(j k)$ direction, with $P_{j k}$ the set of four fixed points between which the D2-brane is "spanned". Furthermore, $\epsilon$ and $\delta_{i}$ are signs, and the configurations of signs $\delta_{i}$ arise from Wilson lines, i.e., not all configurations of signs are allowed. The complete even self-dual charge lattice is spanned by ${ }^{7}$

(I) Nine fractional D4-branes: one has no Wilson line and all twisted charges $+\frac{1}{4}(\epsilon=+1)$; one has no Wilson line and all twisted charges $-\frac{1}{4}(\epsilon=-1)$; the remaining seven fractional D-branes have twisted charge $+\frac{1}{4}$ at the origin, and different choices of Wilson lines.

(II) Nine fractional D0-branes: one sits at the origin and has twisted charge +1 ; one sits at the origin and has twisted charge -1 ; the remaining seven fractional D0-branes have charge +1 and sit at different fixed points.

(III) Six fractional D2-branes, oriented along all $6=\left(\begin{array}{l}4 \\ 2\end{array}\right)$ two-planes, without any Wilson lines (i.e., $\left.\delta_{i}=+1\right)$ and positive twisted charge $(\epsilon=+1)$.

The non-zero entries of the intersection form are

$$
\begin{aligned}
\langle\langle\mathrm{D} 4, \mathrm{U} \mid \mathrm{D} 0, \mathrm{U}\rangle\rangle & =2, \\
\langle\langle\mathrm{D} 2(12), \mathrm{U} \mid \mathrm{D} 2(34), \mathrm{U}\rangle\rangle & =\langle\langle\mathrm{D} 2(13), \mathrm{U} \mid \mathrm{D} 2(42), \mathrm{U}\rangle\rangle \\
& =\langle\langle\mathrm{D} 2(14), \mathrm{U} \mid \mathrm{D} 2(23), \mathrm{U}\rangle\rangle=2, \\
\langle\langle\mathrm{D} *, i \mid \mathrm{D} *, j\rangle\rangle & =-2 \delta_{i j} .
\end{aligned}
$$

It is then straightforward to check that the intersection matrix of the above D-branes has determinant 1, i.e., that these 24 branes generate indeed the full charge lattice.

The RR charges that transform in the $(\mathbf{2}, \mathbf{2})$-representation of $\mathrm{SU}(2)_{\mathrm{L}} \times$ $\mathrm{SU}(2)_{\mathrm{R}}$ and hence span $\Pi$ are precisely those carried by the bulk brane combinations

$$
(\mathrm{D} 0+\mathrm{D} 4),(\mathrm{D} 2(12)+\mathrm{D} 2(34)),(\mathrm{D} 2(13)+\mathrm{D} 2(42)),(\mathrm{D} 2(14)+\mathrm{D} 2(23)),
$$

\footnotetext{
${ }^{7}$ The explicit description of the relevant branes and their intersection matrix is given in the $\mathrm{AT}_{\mathrm{E} X} \mathrm{X}$ source on the arXiv (http://arxiv .org/format/1106.4315v1).
} 
where D2 $(i j)$ denotes the bulk D2-brane oriented along the $(i j)$ direction; this can be deduced from the analysis of [25], see in particular Appendix A.2. The orthogonal complement (with respect to the intersection form) then turns out to be a lattice of rank 20. Upon changing the sign of its quadratic form, this lattice can be embedded into the Leech lattice $\Lambda$. Its orthogonal complement in $\Lambda$ is generated by four vectors $y_{1}, \ldots, y_{4} \in \Lambda$ with $y_{i} \cdot y_{j}=$ $4 \delta_{i j}$. According to our general argument, the group of symmetries $G$ of the model is isomorphic to the group of automorphisms of $\Lambda$ that act trivially on $y_{1}, \ldots, y_{4}$. Since $G$ fixes some vectors of norm 8 in $\Lambda$ (for example, $y_{1}+y_{2}$ ), it must be a subgroup of $\mathbb{Z}_{2}^{12} \rtimes \mathbb{M}_{24}$, see Appendix B.3. A more detailed analysis shows that $G=2^{1+8} \rtimes \mathbb{Z}_{2}^{3}$, thus matching the results from above.

\section{The Gepner model $(1)^{6}$}

Next we consider the $(1)^{6}$ Gepner model which will turn out to realize case (iii) of the Theorem. It is constructed by taking six tensor powers of the $\mathcal{N}=$ 2 minimal model at $k=1$, subject to the $\mathbb{Z}_{3}$ orbifold projection generated by

$$
\bigotimes_{i=1}^{6} \Phi_{m_{i}, s_{i} ; \bar{m}_{i}, \bar{s}_{i}}^{l} \mapsto \mathrm{e}^{\frac{2 \pi \mathrm{i}}{3} \sum_{i} m_{i}} \bigotimes_{i=1}^{6} \Phi_{m_{i}, s_{i} ; \bar{m}_{i}, \bar{s}_{i}}^{l} .
$$

For a short review of Gepner models, as well as an outline of our notations and conventions see Appendix C.

\subsection{The spectrum}

The orbifold theory has an untwisted $\left(\mathcal{H}^{(0)}\right)$ and two twisted $\left(\mathcal{H}^{(1)}\right.$ and $\left.\mathcal{H}^{(-1)}\right)$ sectors, with spectrum

$$
\mathcal{H}^{(n)}=\bigotimes_{i=1}^{6} \mathcal{H}_{l_{i}, m_{i}+n, s_{i}} \otimes \overline{\mathcal{H}}_{l_{i}, m_{i}-n, \bar{s}_{i}} .
$$

Invariance under the orbifold symmetry requires

$$
\sum_{i=1}^{6} m_{i} \equiv 0 \quad \bmod 3
$$

For $k=1$, we may take $l=0$, with $m \in \mathbb{Z} / 6 \mathbb{Z}$ and $s \in \mathbb{Z} / 4 \mathbb{Z}$. The only states that contribute to the elliptic genus are the RR states with $\bar{h}=\frac{1}{4}$. It is easy to see that the condition $\bar{h}=\frac{1}{4}$ together with the $U(1)$-charge integrality 
condition (that follows from orbifold invariance) is only satisfied if the rightmoving ground state is of the form

$$
(0,1,1)^{\otimes 6}, \quad(0,-1,-1)^{\otimes 6}, \quad(0,1,1)^{\otimes 3}(0,-1,-1)^{\otimes 3} .
$$

In the last case, all the 20 different permutations of the factors should be considered. Thus, the RR states for which the right-movers are $\mathrm{R}$ ground states are explicitly

$$
\begin{aligned}
& n=0: \quad \otimes_{i}^{6} \Phi_{1, s_{i} ; 1,1}^{0} \quad \text { (1 state) } \\
& \otimes_{i}^{6} \Phi_{-1, s_{i} ;-1,-1}^{0} \quad \text { (1 state) } \\
& \left.\left(\otimes_{i}^{3} \Phi_{1, s_{i} ; 1,1}^{0}\right) \otimes\left(\otimes_{i}^{3} \Phi_{-1, s_{i} ;-1,-1}^{0}\right) \quad \text { (20 states }\right), \\
& \text { and permutations } \\
& n=1: \quad \otimes_{i}^{6} \Phi_{3, s_{i} ; 1,1}^{0}, \quad \text { (1 state) } \\
& \otimes_{i}^{6} \Phi_{1, s_{i} ;-1,-1}^{0} \quad \text { (1 state) } \\
& \left.\left(\otimes_{i}^{3} \Phi_{3, s_{i} ; 1,1}^{0}\right) \otimes\left(\otimes_{i}^{3} \Phi_{1, s_{i} ;-1,-1}^{0}\right) \quad \text { (20 states }\right), \\
& \text { and permutations } \\
& \begin{array}{rlr}
n=-1: & \otimes_{i}^{6} \Phi_{-1, s_{i} ; 1,1}^{0} & \text { (1 state) } \\
& \otimes_{i}^{6} \Phi_{3, s_{i} ;-1,-1}^{0} \text { (1 state) } \\
& \left(\otimes_{i}^{3} \Phi_{-1, s_{i} ; 1,1}^{0}\right) \otimes\left(\otimes_{i}^{3} \Phi_{3, s_{i} ;-1,-1}^{0}\right) & (20 \text { states }),
\end{array}
\end{aligned}
$$

and permutations

where $s_{i}=1,3$. In each case, states with $s_{i}=1$ and $s_{i}=3, i=1, \ldots, 6$, are mapped into one another under the action of the $\mathcal{N}=2$ algebra of the $i$ th model. The contribution to the elliptic genus of the $\mathcal{N}=2$ representation containing $\otimes_{i}^{6} \Phi_{m_{i}, s_{i} ; \bar{m}_{i}, \bar{s}_{i}}^{0}$ is

$$
\prod_{i}^{6} I_{m_{i}}^{0}(\tau, z) I_{\bar{m}_{i}}^{0}(\bar{\tau}, 0)
$$

where $I_{m}^{l}(\tau, z)$ are the $\mathcal{N}=2$ "characters" that are defined in (C.9). Since

$$
I_{-1}^{0}(\bar{\tau}, 0)=-1, \quad I_{1}^{0}(\bar{\tau}, 0)=1, \quad I_{3}^{0}(\bar{\tau}, 0)=0
$$

we obtain

$$
\phi(\tau, z)=2 \sum_{\substack{m \in \mathbb{Z} / 6 \mathbb{Z} \\ m \text { odd }}} I_{m}^{0}(\tau, z)^{6}-20 \sum_{\substack{m \in \mathbb{Z} / 6 \mathbb{Z} \\ m \text { odd }}} I_{m}^{0}(\tau, z)^{3} I_{m+2}^{0}(\tau, z)^{3},
$$


which reproduces indeed (2.1).

\subsection{Symmetries and twining genera}

Let us first describe the symmetries that preserve the $\mathcal{N}=2$ superconformal symmetry. For $i \in\{1, \ldots, 6\}$, we denote by $e_{i}$ the $\mathbb{Z}_{3}$ symmetry that acts as

$$
e_{i}\left(\bigotimes_{j=1}^{6} \Phi_{m_{j}, s_{j} ; \bar{m}_{j}, \bar{s}_{j}}^{0}\right)=\mathrm{e}^{\frac{2 \pi \mathrm{i} m_{i}}{3}}\left(\bigotimes_{j=1}^{6} \Phi_{m_{j}, s_{j} ; \bar{m}_{j}, \bar{s}_{j}}^{0}\right)
$$

These symmetries generate a group $\mathbb{Z}_{3}^{5}$ (because the product of all of them is the orbifold symmetry, under which all states are invariant by construction). There are also the right-moving analogues $\bar{e}_{i}$, where the phase depends on $\bar{m}_{i}$ instead of $m_{i}$. In addition, we have the quantum symmetry $\mathcal{Q}$ which acts by multiplication by $\mathrm{e}^{\frac{2 \pi \mathrm{in} n}{3}}$ on the $n$th twisted sector $\mathcal{H}^{(n)}$. This gives an additional $\mathbb{Z}_{3}$. Note that in the $n$th twisted sector $\bar{m}_{i}=m_{i}-2 n$, and thus

$$
\bar{e}_{i}=\mathcal{Q} e_{i}
$$

implying that the transformations $\mathcal{Q}, e_{i}, \bar{e}_{i}$ form a group $\mathbb{Z}_{3}^{6}$. Finally, there are permutations of the six factors of $(1)^{6}$, where we define the action of $\pi \in S_{6}$ by

$$
\pi\left(\bigotimes_{i=1}^{6} \Phi_{m_{i}+n, 0 ; m_{i}-n, 0}^{l}\right)=\operatorname{sgn}(\pi)^{n}\left(\bigotimes_{i=1}^{6} \Phi_{m_{\pi(i)}+n, 0 ; m_{\pi(i)}-n, 0}^{l}\right)
$$

with additional signs for $s_{i}, \bar{s}_{i} \neq 0$, given by the usual bosonic/fermionic statistics.

Thus, the group of symmetries preserving the $\mathcal{N}=2$ superconformal symmetry is $\mathbb{Z}_{3} \times \mathbb{Z}_{3}^{5} . S_{6}$. The subgroup preserving the $\mathcal{N}=4$ superconformal algebra is then generated by the transformations that leave the currents $J^{+}$and $J^{-}$of the $\mathcal{N}=4$ superconformal algebra

$$
\left(\Phi_{-2,2 ; 0,0}^{0}\right)^{\otimes 6} \in \mathcal{H}^{(-1)}, \quad\left(\Phi_{2,2 ; 0,0}^{0}\right)^{\otimes 6} \in \mathcal{H}^{(+1)}
$$

invariant. This group has the structure $\mathbb{Z}_{3}^{4} \rtimes A_{6}$ and is generated by ${ }^{8}$

\footnotetext{
${ }^{8}$ We have also checked that these symmetries preserve the spectral flow operators in the RR sector.
} 
(1) The phase transformations

$$
\prod_{i=1}^{6} e_{i}^{n_{i}} \quad \text { with } \quad \sum_{i=1}^{6} n_{i} \equiv 0 \quad \bmod 3
$$

where the constraint assures invariance of the currents. Because of the orbifold invariance relation $\prod_{i=1}^{6} e_{i}=1$, they generate the normal subgroup $\mathbb{Z}_{3}^{4}$.

(2) The even permutations, since the odd permutations act on the states (4.11) with a minus sign. The even permutations form the alternating group $A_{6}$.

The resulting group is the semidirect product $\mathbb{Z}_{3}^{4} \rtimes A_{6}$, where we have the obvious action $\pi\left(\prod_{i=1}^{6} e_{i}^{n_{i}}\right)=\prod_{i=1}^{6} e_{\pi(i)}^{n_{i}}$ of $A_{6}$ on the generators of $\mathbb{Z}_{3}^{4}$. The $(1)^{6}$ Gepner model is therefore an example of case (iii) of the Theorem.

With this description it is now straightforward to calculate the corresponding twining genera, and for the convenience of the reader we have collected them in table 1 . For example, we have for the phase transformation $e_{1} e_{2} e_{3}$

$$
\begin{aligned}
\phi_{e_{1} e_{2} e_{3}}(\tau, z) & =2 \sum_{m=-1,1,3} I_{m}^{6}-\left(2+9 \mathrm{e}^{2 \pi \mathrm{i} / 3}+9 \mathrm{e}^{-2 \pi \mathrm{i} / 3}\right) \sum_{m=-1,1,3} I_{m}^{3} I_{m+2}^{3} \\
& =2 \sum_{m=-1,1,3} I_{m}^{6}+7 \sum_{m=-1,1,3} I_{m}^{3} I_{m+2}^{3}=-\frac{1}{2} \phi_{1 \mathrm{~A}}+\frac{3}{2} \phi_{3 \mathrm{~A}} \equiv \hat{\phi}_{3 \mathrm{a}}
\end{aligned}
$$

which corresponds to the fourth line of the table. The other twining genera that do not directly agree with $\mathbb{M}_{24}$ twining genera - since we are in case (iii) of the Theorem, there is no reason to expect any $\mathbb{M}_{24}$ twining genera - are

$$
\begin{aligned}
& \hat{\phi}_{6 \mathrm{a}}(\tau, z)=\frac{1}{2}\left(\phi_{2 \mathrm{~A}}(\tau, z)+\phi_{6 \mathrm{~A}}(\tau, z)\right), \\
& \hat{\phi}_{9 \mathrm{a}}(\tau, z)=\frac{1}{2}\left(\phi_{3 \mathrm{~A}}(\tau, z)+\phi_{3 \mathrm{~B}}(\tau, z)\right),
\end{aligned}
$$

$$
\hat{\phi}_{9 \mathrm{~b}}(\tau, z)=\frac{1}{4}\left[\phi_{0,1}(\tau, z)+6\left(2 \psi^{(3)}(\tau)+3 \psi^{(9)}(\tau)+3 E_{2}^{(9)}(\tau)\right) \phi_{-2,1}(\tau, z)\right],
$$

where $\phi_{0,1}$ and $\phi_{-2,1}$ are the standard Jacobi forms of $\operatorname{SL}(2, \mathbb{Z})$ of index one and weight 0 and -2 , respectively, and $\left\{\psi^{(3)}, \psi^{(9)}, E_{2}^{(9)}\right\}$ form a basis 
Table 1: Twining genera of the $(1)^{6}$ model. The symmetry generators have been labelled by the structure of the permutations $\{i, j, k, l, m, n\}$ of the minimal models and the individual phase shifts $e_{i}^{n_{i}} e_{j}^{n_{j}} e_{k}^{n_{k}} e_{l}^{n_{l}} e_{m}^{n_{m}} e_{n}^{n_{n}}$. The multiplicity (labelled by \#) is the number of "independent" generators within each class of symmetries which are not identified through the action of the orbifold. The order of each generator is denoted by $N$ and $\operatorname{Tr}_{24}$ gives the trace over the 24-dimensional representation. Finally, the twining genera $\hat{\phi}_{3 \mathrm{a}}, \hat{\phi}_{6 \mathrm{a}}$ and $\hat{\phi}_{9 \mathrm{ab}}$ are not Mathieu twining genera and are defined in the main body of the text.

\begin{tabular}{|c|l||r|r|r|r|}
\hline \multicolumn{2}{|c||}{ Symmetry } & \multicolumn{3}{c|}{ Properties } \\
\hline \hline Permutations & Conditions on phases & $N$ & $\#$ & $\operatorname{Tr}_{24}$ & $\phi_{g}$ \\
\hline \hline$i j k l m n$ & $n_{i}=n_{j}=n_{k}=n_{l}=m_{m}=n_{n}=0$ & 1 & 1 & 24 & $\phi_{1 \mathrm{~A}}$ \\
\hline$(i j)(k l) m n$ & $n_{i}+n_{j}=n_{k}+n_{l}=n_{m}=n_{n}=0$ & 2 & 405 & 8 & $\phi_{2 \mathrm{~A}}$ \\
\hline $\begin{array}{c}i j k l m n \\
(i j k) l m n\end{array}$ & $n_{i}=2, n_{j}=1, n_{k}+n_{l}+n_{m}+n_{m}=0$ & & & & \\
$(i j k)(\operatorname{lmn})$ & $n_{i}+n_{j}+n_{k}=0, n_{l}+n_{m}+n_{n}=0$ & 3 & 2220 & 6 & $\phi_{3 \mathrm{~A}}$ \\
\hline$i j k l m n$ & $n_{i}+n_{j}+n_{k}=0, n_{l}+n_{m}+n_{n}=0$ & & & & \\
\hline$(i j k l)(m n)$ & $n_{i}=n_{j}=n_{k}=1, n_{l}=n_{m}=n_{n}=0$ & 3 & 20 & -3 & $\hat{\phi}_{3 \mathrm{a}}$ \\
\hline$(i j k l m) n$ & $n_{i}+n_{j}+n_{k}+n_{l}+n_{m}+n_{n}=0$ & 4 & 7290 & 4 & $\phi_{4 \mathrm{~B}}$ \\
\hline $\begin{array}{c}(i j)(k l) m n \\
(i j)(k l) m n\end{array}$ & $n_{i}+n_{j}=n_{k}+n_{l}=0, n_{m}=1, n_{n}=2$ & & & & \\
$(i j)(k l) m n$ & $n_{i}+n_{j}=n_{k}+n_{l}=1, n_{m}=1, n_{n}=0$ & & & & \\
$(i j)(k l) m n$ & $n_{i}+n_{j}=n_{k}+n_{l}=2, n_{m}=2, n_{n}=0$ & 6 & 1620 & 2 & $\phi_{6 \mathrm{~A}}$ \\
$(i j)(k l) m n$ & $n_{i}+n_{j}=1, n_{k}+n_{l}=0, n_{m}+n_{n}=2$ & & & & \\
\hline$(i j)(k l) m n$ & $n_{i}+n_{j}=2, n_{k}+n_{l}=0, n_{m}=1, n_{n}=0$ & 6 & 1620 & 5 & $\hat{\phi}_{6 \mathrm{a}}$ \\
\hline$(i j k)(\operatorname{lmn})$ & $n_{i}+n_{j}+n_{k}=2, n_{l}+n_{m}+n_{n}=1$ & 9 & 3240 & 3 & $\hat{\phi}_{9 \mathrm{a}}$ \\
\hline $\begin{array}{c}(i j k) \operatorname{lmn} \\
(i j k) \operatorname{lmn}\end{array}$ & $n_{i}+n_{j}+n_{k}=2, n_{l}+n_{m}+n_{n}=1$ & & \multirow{2}{*}{1080} & 3 & $\hat{\phi}_{9 \mathrm{~b}}$ \\
\hline
\end{tabular}

of weight two modular forms of $\Gamma_{0}(9)$ (for more information and explicit definitions see [5]).

\subsection{The D-brane charge lattice}

As before, we can also determine the symmetry group of this model from an analysis of the D-brane charge lattice. The construction of Gepner model D-branes is standard, and is briefly sketched in Appendix C. The tensor 
product A-type branes with $L_{i}=S_{i}=0$ and $M_{1}, \ldots, M_{6} \in \mathbb{Z}_{6}$ generate a charge lattice of rank 22 with signature $(2,20)$. This is as expected, since the A-type tensor product branes only couple to the $22 \mathrm{RR}$ ground states in the untwisted sector.

The other charges are carried by B-type permutation branes of the type described in (C.25). As we vary $M_{1}, \ldots, M_{6}, \hat{M} \in \mathbb{Z}_{6}$, the intersection form of the B-type branes gives a matrix of rank 10 with signature $(2,8)$. Again, this is what we expect since these permutation branes couple to the $2 \mathrm{RR}$ ground states in the twisted sectors $n= \pm 1$, and to the $8 \mathrm{RR}$ ground states in the untwisted sector with $m_{1}=-m_{2}, m_{3}=-m_{4}$, and $m_{5}=-m_{6}$.

In order to obtain the full charge lattice we have to combine these two constructions; for example, a set of 22 A-type D-branes with $L_{i}=0=S_{i}$, $i=1, \ldots, 6$ and suitable values for $M_{1}, \ldots, M_{6}$, and two B-type D-branes with $L_{i}=M_{i}=0, S_{i}=0$ and $\hat{M}= \pm 2$ generate the full unimodular lattice $\Gamma^{4,20}$ (see the LATEX source code for details).

Next, we denote that four $\mathrm{RR}$ ground states in the $(\mathbf{2}, \mathbf{2})$ representation of $\mathrm{SU}(2)_{\mathrm{L}} \times \mathrm{SU}(2)_{\mathrm{R}}$ according to their $J_{0}^{3}, \tilde{J}_{0}^{3}$ charges as $\Phi_{1, \overline{1}}, \Phi_{1,-\overline{1}}, \Phi_{-1, \overline{1}}$, and $\Phi_{-1,-\overline{1}}$, Let us consider the sublattice $\left(\Gamma^{4,20}\right)^{\perp}$ of D-branes that are neutral under these four states. The 22 A-type branes generate the sublattice of D-branes that are neutral with respect to $\Phi_{1,-\overline{1}}$ and $\Phi_{-1, \overline{1}}$, while their charge with respect to $\Phi_{1, \overline{1}}$ and $\Phi_{-1,-\overline{1}}$ is given by

$$
\begin{aligned}
\left.\left.Q_{\Phi_{1, \overline{1}}}\left(\| 0, M_{i}, 0\right\rangle\right\rangle_{\mathrm{A}}\right) & =\frac{1}{3} \mathrm{e}^{\frac{\pi \mathrm{i}}{3} \sum_{i} M_{i}}, \\
\left.\left.Q_{\Phi_{-1,-\overline{1}}}\left(\| 0, M_{i}, 0\right\rangle\right\rangle_{\mathrm{A}}\right) & =\frac{1}{3} \mathrm{e}^{-\frac{\pi \mathrm{i}}{3} \sum_{i} M_{i}} .
\end{aligned}
$$

With the redefinition

$$
\Phi^{a}=3\left(\Phi_{1, \overline{1}}+\Phi_{-1,-\overline{1}}\right), \quad \Phi^{b}=2 \sqrt{3} \mathrm{i}\left(\Phi_{1, \overline{1}}-\Phi_{-1,-\overline{1}}\right),
$$

the states $\Phi^{a}, \Phi^{b}$ correspond to elements of the dual lattice $\left(\Gamma^{4,20}\right)^{*} \cong \Gamma^{4,20}$ so that the sublattice generated by A-type D-branes and orthogonal to these elements has maximal rank,

$$
\operatorname{rk}\left(\Gamma^{4,20}\right)^{\perp}=20
$$

The discriminant group of this 20-dimensional lattice is $\mathbb{Z}_{9} \times \mathbb{Z}_{3}^{2}$, and its discriminant form is the same as the one of the S-lattice $2^{9} 3^{6}$ (see 
Appendix B.3), whose quadratic form is

$$
Q_{2^{9} 3^{6}}=\left(\begin{array}{rrrr}
4 & 1 & 1 & -2 \\
1 & 4 & 1 & -2 \\
1 & 1 & 4 & 1 \\
-2 & -2 & 1 & 4
\end{array}\right)
$$

By the general lattice gluing procedure [19], it follows that the S-lattice $2^{9} 3^{6}$ and $\left(\Gamma^{4,20}\right)^{\perp}(-1)$ are orthogonal sublattices of a positive definite even unimodular lattice of rank 24. This procedure also provides explicitly the quadratic form for this unimodular lattice. With the help of some computer algorithm we have shown that the resulting lattice has no vectors of norm 2 , thus proving that it is indeed the Leech lattice $\Lambda$. All sublattices of $\Lambda$ with quadratic form (4.23) are related to the S-lattice $2^{9} 3^{6}$ by some Leech lattice automorphism, and the pointwise stabilizer of each of them is the group $G=\mathbb{Z}_{3}^{4} \rtimes A_{6}[26]$, thus matching the results from the previous subsection.

\section{The $(2)^{4}$ model}

Our last example is the "quartic" model $(2)^{4}$ which is constructed by taking a $\mathbb{Z}_{4}$ orbifold of four tensor powers of the $\mathcal{N}=2$ minimal model of level $k=2$. Our notation and conventions are the same as in the previous section and are again summarized in Appendix C.

\subsection{The spectrum}

For the $\mathbb{Z}_{4}$ orbifold we have in addition to the untwisted sector $\mathcal{H}^{(0)}$ three twisted sectors $(n=1,2,3)$, with spectrum

$$
\mathcal{H}^{(n)}=\bigotimes_{i=1}^{4} \mathcal{H}_{l_{i}, m_{i}+n, s_{i}} \otimes \overline{\mathcal{H}}_{l_{i}, m_{i}-n, \bar{s}_{i}}
$$

where invariance under the $\mathbb{Z}_{4}$-orbifold enforces $\sum_{i=1}^{4} m_{i} \equiv 0 \bmod 4$. At $k=2$ there are $\operatorname{six} \mathcal{N}=2$ R-sector representations, which we may label by $(l=0, m= \pm 1, \pm 3)$ and $(l=1, m=0,2)$. For the elliptic genus we are again only interested in those $\mathrm{RR}$ states for which the right-moving states are ground states with $\bar{h}=\frac{1}{4}$. The relevant coset representations are (compare 
also $[25,27])$

$$
\begin{gathered}
(0,1,1)^{\otimes 4}, \quad(1,2,1)^{\otimes 4}, \quad(0,-1,-1)^{\otimes 4}, \\
(0,1,1)^{\otimes 2} \otimes(0,-1,-1)^{\otimes 2}, \quad(0,1,1) \otimes(0,-1,-1) \otimes(1,2,1)^{\otimes 2},
\end{gathered}
$$

where in the second line all 6 and 12 permutations are included, respectively. Explicitly, the RR states that can contribute to the elliptic genus are thus of the form

$$
\begin{array}{llll}
n=0: & n=1: & n=2: & n=3: \\
\otimes_{i}^{4} \Phi_{1, s_{i} ; 1,1}^{0} & \otimes_{i}^{4} \Phi_{3, s_{i} ; 1,1}^{0} & \otimes_{i}^{4} \Phi_{-3, s_{i} ; 1,1}^{0} & \otimes_{i}^{4} \Phi_{-1, s_{i} ; 1,1}^{0} \\
\otimes_{i}^{4} \Phi_{2, s_{i} ; 2,1}^{1} & \otimes_{i}^{4} \Phi_{0, s_{i} ; 2,1}^{1} & \otimes_{i}^{4} \Phi_{-2, s_{i} ; 2,1}^{1} & \otimes_{i}^{4} \Phi_{0, s_{i} ; 2,1}^{1} \\
\otimes_{i}^{4} \Phi_{-1, s_{i} ;-1,-1}^{0} & \otimes_{i}^{4} \Phi_{1, s_{i} ;-1,-1}^{0} & \otimes_{i}^{4} \Phi_{3, s_{i} ;-1,-1}^{0} & \otimes_{i}^{4} \Phi_{-3, s_{i} ;-1,-1}^{0} .
\end{array}
$$

In addition, there are the states (again written in the order $n=0, n=1$, $n=2$, and $n=3$ )

$$
\begin{array}{ll}
\left(\otimes_{i}^{2} \Phi_{1, s_{i} ; 1,1}^{0}\right) \otimes\left(\otimes_{i}^{2} \Phi_{-1, s_{i} ;-1,-1}^{0}\right) & \left(\otimes_{i}^{2} \Phi_{3, s_{i} ; 1,1}^{0}\right) \otimes\left(\otimes_{i}^{2} \Phi_{1, s_{i} ;-1,-1}^{0}\right) \\
\left(\otimes_{i}^{2} \Phi_{-3, s_{i} ; 1,1}^{0}\right) \otimes\left(\otimes_{i}^{2} \Phi_{3, s_{i} ;-1,-1}^{0}\right) & \left(\otimes_{i}^{2} \Phi_{-1, s_{i} ; 1,1}^{0}\right) \otimes\left(\otimes_{i}^{2} \Phi_{-3, s_{i} ;-1,-1}^{0}\right),
\end{array}
$$

where in each case there are 6 different permutations, as well as the states

$$
\begin{aligned}
& \left(\otimes_{i}^{2} \Phi_{2, s_{i} ; 2,1}^{1}\right) \otimes \Phi_{-1, s_{i} ;-1,-1}^{0} \otimes \Phi_{1, s_{i} ; 1,1}^{0} \\
& \left(\otimes_{i}^{2} \Phi_{0, s_{i} ; 2,1}^{1}\right) \otimes \Phi_{1, s_{i} ;-1,-1}^{0} \otimes \Phi_{3, s_{i} ; 1,1}^{0} \\
& \left(\otimes_{i}^{2} \Phi_{-2, s_{i} ; 2,1}^{1}\right) \otimes \Phi_{3, s_{i} ;-1,-1}^{0} \otimes \Phi_{-3, s_{i} ; 1,1}^{0} \\
& \quad\left(\otimes_{i}^{2} \Phi_{0, s_{i} ; 2,1}^{1}\right) \otimes \Phi_{-3, s_{i} ;-1,-1}^{0} \otimes \Phi_{-1, s_{i} ; 1,1}^{0}
\end{aligned}
$$

where now there are 12 different permutations each. Again, the states with $s_{i}=1,3$ are mapped into one another under the action of the $\mathcal{N}=2$ algebra of the $i$ th model. Since

$$
I_{ \pm 1}^{0}(\bar{\tau}, 0)= \pm 1, \quad I_{ \pm 3}^{0}(\bar{\tau}, 0)=0, \quad I_{0}^{1}(\bar{\tau}, 0)=0, \quad I_{ \pm 2}^{1}(\bar{\tau}, 0)= \pm 1
$$

the total contribution to the elliptic genus is then

$$
\phi=\sum_{\substack{m \in \mathbb{Z} / 8 \mathbb{Z} \\ m \text { odd }}}\left[2\left(I_{m}^{0}\right)^{4}+6\left(I_{m}^{0}\right)^{2}\left(I_{m-2}^{0}\right)^{2}-12 I_{m}^{0} I_{m+2}^{0}\left(I_{m+3}^{1}\right)^{2}+\left(I_{m+1}^{1}\right)^{4}\right],
$$

which agrees indeed with (2.1). 


\subsection{Symmetries and twining genera}

In the quartic $(2)^{4}$ model the currents $J^{ \pm}$of the left- and right-moving $\mathcal{N}=4$ superconformal algebra are given by

$$
J^{ \pm}=(0, \pm 2,2)^{\otimes 4} \otimes \overline{(0,0,0)}^{\otimes 4}, \quad \text { and } \quad \bar{J}^{ \pm}=(0,0,0)^{\otimes 4} \otimes \overline{(0, \pm 2,2)}^{\otimes 4} .
$$

The symmetries that leave these currents invariant are

(1) Phase shifts, which are generated by

$$
\prod_{i=1}^{4} e_{i}^{a_{i}}\left(\mathcal{Q}^{2}(-1)^{F_{\mathrm{s}}}\right)^{\frac{A}{2}}, \quad A=\sum_{i=1}^{4} a_{i} \quad \text { with } A \equiv 0 \bmod 2 .
$$

Here each $e_{i}^{a_{i}}$ acts as

$$
e_{i}^{a_{i}}: \Phi_{m_{i}, s_{i} ; \bar{m}_{i}, \bar{s}_{i}}^{\ell} \longmapsto \mathrm{e}^{\frac{2 \pi \mathrm{i} m_{i} a_{i}}{4}} \Phi_{m_{i}, s_{i} ; \bar{m}_{i}, \bar{s}_{i}}^{\ell},
$$

$\mathcal{Q}$ is the quantum symmetry of the Gepner orbifold (that acts as a phase $\mathrm{e}^{\frac{\pi \mathrm{in} n}{2}}$ on the states of the $n$th twisted sector), and $(-1)^{F_{\mathrm{s}}}$ is the left-moving spacetime fermion number operator that acts as $+1(-1)$ on the left-moving NS (R) sector. (The inclusion of $(-1)^{F_{\mathrm{s}}}$ is required in order to preserve the spectral flow operators in the RR sector.) Taking into account the overall $\mathbb{Z}_{4}$ invariance coming from the Gepner orbifold, these phase shifts generate the group $\mathbb{Z}_{4}^{2} \times \mathbb{Z}_{2}$.

(2) The permutations

$$
\pi\left(\otimes_{i=1}^{4} \Phi_{m_{i}+n, 0 ; m_{i}-n, 0}^{l}\right)=\operatorname{sgn}(\pi)^{n} \mathcal{Q}^{\operatorname{sgn}(\pi)-1}\left(\otimes_{i=1}^{4} \Phi_{m_{\pi(i)}+n, 0 ; m_{\pi(i)}-n, 0}^{l}\right) .
$$

They generate the symmetric group $S_{4}$.

These symmetries generate the group $\left(\mathbb{Z}_{2} \times \mathbb{Z}_{4}^{2}\right) \rtimes S_{4}$, thus realizing case (i) of the Theorem with $G^{\prime}=\mathbb{Z}_{2}^{3}$ and $G^{\prime \prime}=\mathbb{Z}_{2}^{2} . S_{4}$. Here $G^{\prime} \subset \mathbb{Z}_{2}^{11}$ is generated by the phases $e_{1}^{2} e_{2}^{2}, e_{2}^{2} e_{3}^{2}$ and $e_{3}^{2} \mathcal{Q}^{2}(-1)^{F_{s}}$, while $G^{\prime \prime} \subset \mathbb{M}_{24}$ is generated by the permutations, giving the $S_{4}$ factor, as well as by the phases $e_{1} e_{2} \mathcal{Q}^{2}(-1)^{F_{s}}$ and $e_{2}^{3} e_{3}^{3} \mathcal{Q}^{2}(-1)^{F_{s}}$, giving the $\mathbb{Z}_{2}^{2}$ factor. ${ }^{9}$

\footnotetext{
${ }^{9}$ Note that these last two phases are order 2 only after taking the quotient by $G^{\prime}$; in fact, their squares are non-trivial elements in $G^{\prime}$, so that as elements of the whole group $G$ they are order 4 .
} 
Table 2: The twining genera of the $(2)^{4}$ model. Here the symmetries have been labelled by the structure of the permutations of $\{i, j, k, l\}$, the phase shifts of the individual symmetries $e_{i}^{n_{i}} e_{j}^{n_{j}} e_{k}^{n_{k}} e_{l}^{n_{l}}$, the power of the operator $Q^{n_{Q}}$ and the spacetime fermion number $(-1)^{n_{F} F_{\mathrm{s}}}$. The remaining part of the notation is the same as for table 1.

\begin{tabular}{|c|l|c|c||c|c|c|c|}
\hline \multicolumn{2}{|c|}{ Symmetry } & \multicolumn{3}{|c|}{ Properties } \\
\hline \hline Permutations & Conditions on phases & $n_{Q}$ & $n_{F}$ & $N$ & $\#$ & $\operatorname{Tr}_{24}$ & $\phi_{g}$ \\
\hline \hline$i j k l$ & $n_{i}=n_{j}=n_{k}=n_{l}=0$ & 0 & 0 & 1 & 1 & 24 & $\phi_{1 \mathrm{~A}}$ \\
\hline$i j k l$ & $n_{i}=n_{j}=2, n_{k}+n_{l}=0$ & 0 & 0 & & & & \\
$(i j)(k l)$ & $n_{i}+n_{j}=n_{k}+n_{l} \in\{0,2\}$ & 0 & 0 & & & & \\
$(i j) k l$ & $n_{i}+n_{j}=n_{k}+n_{l}=0, n_{k, l} \in\{0,2\}$ & 2 & 0 & 2 & 79 & 8 & $\phi_{2 \mathrm{~A}}$ \\
$(i j) k l$ & $n_{i}+n_{j}=0, n_{k}+n_{l}=2, n_{k, l} \in\{0,2\}$ & 0 & 1 & & & & \\
$i j k l$ & $n_{i}+n_{j}+n_{k}+n_{l}=2, n_{i, j, k, l} \in\{0,2\}$ & 2 & 1 & & & & \\
\hline$(i j)(k l)$ & $n_{i}+n_{j}=n_{k}+n_{l} \in\{1,3\}$ & 2 & 1 & 2 & 24 & 0 & $\phi_{2 \mathrm{~B}}$ \\
\hline$(i j k) l$ & $n_{i}+n_{j}+n_{k}+n_{l}=0$ & 0 & 0 & 3 & 128 & 6 & $\phi_{3 \mathrm{~A}}$ \\
\hline$i j k l$ & $n_{i}=n_{j} \in\{1,3\}, n_{k}=n_{l}=0$ & 2 & 1 & 4 & 6 & -4 & $\phi_{4 a}$ \\
\hline$i j k l$ & $n_{i}=1, n_{j}+n_{k}+n_{l}=3$ & 2 & 0 & & & & \\
$(i j k l)$ & $n_{i}+n_{j}+n_{k}+n_{l}=0$ & 0 & 0 & & & & \\
$(i j)(k l)$ & $n_{i}=n_{j}=1, n_{k}+n_{l}=2$ & 0 & 1 & 4 & 306 & 4 & $\phi_{4 \mathrm{~B}}$ \\
$(i j) k l$ & $n_{i}+n_{j}+n_{k}+n_{l}=2, n_{i, j} \in\{1,3\}$ & 0 & 1 & & & & \\
$(i j) k l$ & $n_{i}+n_{j}+n_{k}+n_{l}=2, n_{k, l} \in\{1,3\}$ & 0 & & & \\
$(i j) k l$ & $n_{i}+n_{j}=n_{k}+n_{l}=0, n_{i, j} \in\{1,3\}$ & 2 & 0 & & & & \\
$(i j) k l$ & $n_{i}+n_{j}=n_{k}+n_{l}=0, n_{k, l} \in\{1,3\}$ & 2 & 0 & & & & \\
$i j k l$ & $n_{i}=1, n_{j}+n_{k}+n_{l}=1$ & 2 & 1 & & & & \\
\hline$(i j k) l$ & $n_{i}+n_{j}+n_{k}+n_{l}=2$ & 2 & 1 & 6 & 128 & 2 & $\phi_{6 \mathrm{~A}}$ \\
\hline$(i j) k l$ & $n_{i}+n_{j}=1, n_{k}+n_{l}=3$ & 0 & 8 & 96 & 2 & $\phi_{8 \mathrm{~A}}$ \\
\hline
\end{tabular}

We can also calculate the associated twining genera, and our results are collected in Table 2. Again, we see that some of the generators in $G^{\prime}$ lead to the twining genus

$$
\hat{\phi}_{4 a}=-\frac{1}{2}\left(\phi_{1 \mathrm{~A}}-\phi_{2 \mathrm{~A}}-2 \phi_{4 \mathrm{~B}}\right)
$$

that does not coincide with any twining genus of $\mathbb{M}_{24}$. 


\subsection{The D-brane charge lattice}

The derivation of a set of D-branes generating the lattice of $\mathrm{RR}$ charges is analogous to the construction for the $(1)^{6}$ model. The A-type tensor product branes (see Appendix C.2) are now only charged under the $21 \mathrm{RR}$ ground states in the untwisted sector. The remaining charges can be accounted for in terms of B-type permutation branes. Taking 21 A-type branes with $L_{i}=$ $S_{i}=0$ and suitable combinations for $M_{i}$, as well as three B-type permutation branes with $L_{i}=M_{i}=S_{i}=0$ and suitable values of $\hat{M}$ leads indeed to the full charge lattice $\Gamma^{4,20}$, i.e., the resulting intersection form has determinant one. ${ }^{10}$

The sublattice $\left(\Gamma^{4,20}\right)^{\perp}$ of D-branes that are neutral with respect to the $R R$ ground states in the $(\mathbf{2}, \mathbf{2})$ representation of $\mathrm{SU}(2)_{\mathrm{L}} \times \mathrm{SU}(2)_{\mathrm{R}}$ has maximal rank 20. Upon changing the sign of its quadratic form, it can be embedded into the Leech lattice $\Lambda$, and its orthogonal complement $\Lambda^{G}$ has quadratic form

$$
\left(\begin{array}{rrrr}
6 & 2 & 0 & 0 \\
2 & 4 & -2 & 4 \\
0 & -2 & 6 & -4 \\
0 & 4 & -4 & 8
\end{array}\right)
$$

Since $\Lambda^{G}$ contains a vector of norm 8 , its point-wise stabilizer must be a subgroup of $\mathbb{Z}_{2}^{12} \rtimes \mathbb{M}_{24}$ (see Appendix B.3). More precisely, we have shown that the stabilizer turns out to be isomorphic to $G=\left(\mathbb{Z}_{2} \times \mathbb{Z}_{4}^{2}\right) \rtimes S_{4}$, which is the group of symmetries we have found in the previous subsection.

\section{Conclusions}

In this paper, we have shown that the symmetries of a non-linear $\sigma$-model on $\mathrm{K} 3$ that preserve the $\mathcal{N}=(4,4)$-superconformal algebra as well as the spectral flow operators in the RR sector, form a subgroup of the Conway group $\mathrm{Co}_{1}$. This provides a stringy analogue of the Mukai theorem in algebraic geometry that shows that the symplectic automorphisms of any K3 form a subgroup of the Mathieu group $\mathbb{M}_{23}$. The specific subgroups that can actually arise in our case are spelled out in the Theorem stated in the Introduction.

\footnotetext{
${ }^{10}$ See the $\mathrm{LT}_{\mathrm{E} X}$ source code at http://arxiv.org/format/1106.4315v1 for details; there we also give further details about the sublattice $\left(\Gamma^{4,20}\right)^{\perp}$ and its embedding into the Leech lattice.
} 
Our result is somewhat unexpected in view of the recent observation of [1], relating the elliptic genus of K3 to the Mathieu group $\mathbb{M}_{24}$. In particular, it follows from our Theorem (as well as the explicit examples) that the symmetries of a given K3 model are not, in general, subgroups of $\mathbb{M}_{24} \cdot{ }^{11}$ As a consequence, their twining genera do not, in general, agree with those appearing in the context of Mathieu moonshine [3-6], and we have seen explicit examples of this. In particular, this therefore means that the naive idea that $\mathbb{M}_{24}$ arises as the "union" of all symmetries from different points in moduli space needs to be refined.

At least on the face of it, our Theorem seems to suggest that the elliptic genus of $\mathrm{K} 3$ could exhibit some sort of moonshine based on $\mathrm{Co}_{1}$ or $\mathrm{Co}_{2}$, but we have seen no evidence of this since the dimensions of their representations do not match the coefficients of the elliptic genus. It is intriguing that a connection between $\mathrm{Co}_{1}$ and the BKM algebras arising in $\mathbb{T}^{6}$-compactifications of the heterotic string has recently been observed in [28]; given that the heterotic string on $\mathbb{T}^{6}$ is dual to type IIA on $K 3 \times \mathbb{T}^{2}$, this could be related to our findings.

Our analysis also provides useful tools for the general understanding of non-linear $\sigma$-models on K3. For example, our Theorem suggests the existence of models with some large symmetry groups, and gives precise predictions for their lattice of D-brane charges. These predictions were nicely verified in the three examples we considered. In particular, we showed that the $(1)^{6}$ Gepner model realizes case (iii) of the Theorem. Some preliminary investigations suggest that the groups described in case (iv) might be realized in terms of $\mathbb{T}^{4} / \mathbb{Z}_{3}$ torus orbifolds for different choices of metric and B-field, while it is more difficult to guess which model realizes case (ii). For case (i), the Theorem predicts the existence of a model with symmetry group $\mathbb{Z}_{2}^{8} \rtimes \mathbb{M}_{20}$, that might correspond to a certain $\mathbb{T}^{4} / \mathbb{Z}_{2}$ orbifold.

On more general grounds, the sublattice of D-branes that are neutral under the RR ground states in the $(\mathbf{2}, \mathbf{2})$ representation of $\mathrm{SU}(2)_{\mathrm{L}} \times \mathrm{SU}(2)_{\mathrm{R}}$ is, in a certain sense, the stringy analogue of the Picard lattice in algebraic geometry. Since the groups of symmetries have a genuine action on this sublattice, it would be interesting to understand for which models this lattice has maximal rank 20. For example, one can show that for a Gepner model of type $\left(k_{1}\right) \cdots\left(k_{r}\right)$, a necessary condition for this to happen is that the greatest common divisor $\operatorname{gcd}\left(k_{1}+2, k_{2}+2, \ldots\right)$ of their shifted levels is 3,4 or 6 .

\footnotetext{
${ }^{11}$ Apparently this was also independently noted by the authors of [1]; we thank
} Yuji Tachikawa for discussions about this point. 


\section{Acknowledgments}

We thank Michael Douglas, Martin Fluder, Terry Gannon, Daniel Persson and Yuji Tachikawa for useful discussions and correspondences. The research of MRG was supported by the Swiss National Science Foundation.

\section{Appendix A. Notation and mathematical background}

\section{Appendix A.1. Group theory}

Let us give a brief summary of our conventions regarding finite groups [20].

$A \times B$ The direct product of the groups $A$ and $B$.

$N \rtimes H$ The semidirect product of $H$ acting on the normal subgroup $N$.

N.Q A group $G$ having $N$ as a normal subgroup such that $G / N \cong Q$. (This notation includes the direct and semidirect product as special subcases.)

$\mathbb{Z}_{n} \quad$ Cyclic group of order $n$.

$p_{+}^{1+2 n} \quad$ Extra-special group of order $p^{1+2 n}$ (we will always omit the plus in our notation). For a prime $p$ and positive integer $n$, $p^{1+2 n}$ is the extension of $\mathbb{Z}_{p}^{2 n}$ by a central element $z$ of order $p$. It is generated by $2 n$ elements $x_{1}, \ldots, x_{n}, y_{1}, \ldots, y_{n}$ of order $p$, with $x_{i} x_{j}=x_{j} x_{i}, y_{i} y_{j}=y_{j} y_{i}$ and $\prod x_{i}^{r_{i}} \prod y_{j}^{s_{j}}=$ $z^{r \cdot s} \prod y_{j}^{s_{j}} \prod x_{i}^{r_{i}}$.

$S_{n} \quad$ Group of permutations of $n$ elements (symmetric group).

$A_{n} \quad$ Group of even permutations of $n$ elements (alternating group).

$\mathbb{M}_{24}$ The largest Mathieu group, a sporadic simple group of order $2^{10} \cdot 3^{3} \cdot 5 \cdot 7 \cdot 11 \cdot 23=244823040$. It can be described as a group of permutations of 24 elements. More precisely, it is the subgroup of $S_{24}$ that preserves the (extended) binary Golay code. This group has 26 conjugacy classes. 
$\mathbb{M}_{23}$ The subgroup of $\mathbb{M}_{24}$ that, in its representation as a permutation of 24 elements, fixes one element.

$\mathrm{Co}_{0}$ The Conway group is the automorphism group $\operatorname{Aut}(\Lambda)$ of the Leech lattice. It is an extension of $\mathrm{Co}_{1}$ by the central $\mathbb{Z}_{2}$ that flips the sign of all vectors in $\Lambda$.

$\mathrm{Co}_{1}$ A sporadic simple group of order $2^{21} \cdot 3^{9} \cdot 5^{4} \cdot 7^{2} \cdot 11 \cdot 13$. $23=4157776806543360000$.

$\mathbb{Z}_{2}^{12} \rtimes \mathbb{M}_{24} \quad$ A maximal subgroup of $\operatorname{Aut}(\Lambda)$. It fixes a set of 24 mutually orthogonal vectors $x_{1}, \ldots, x_{24} \in \Lambda$ of norm 8 $\left(x_{i} \cdot x_{j}=8 \delta_{i j}\right)$, up to signs. Each element of the normal subgroup $\mathbb{Z}_{2}^{12}$ changes the sign of $n$ of these 24 vectors, with $n=0,8,12,16,24$. This description establishes a one to one correspondence of $\mathbb{Z}_{2}^{12}$ with the (extended) binary Golay code [19]. The subgroup $\mathbb{M}_{24}$ acts by permutations of $x_{1}, \ldots, x_{24}$.

\section{Appendix A.2. Lattices}

For any lattice $L$, we denote by $L^{*}$ its dual lattice and by $L(n), n \in \mathbb{R}$, the lattice obtained from $L$ by multiplying the quadratic form by $n$. If $L$ is integral, then $L \subseteq L^{*}$, and the finite abelian group $A_{L}=L^{*} / L$ is called the discriminant group. We denote by $l(L)$ the minimal number of generators of $A_{L}$ (notice that $l(L) \leq \operatorname{rk} L$, with $\mathrm{rk} L$ the rank of the lattice ).

Let $A_{L}$ be the discriminant group of an even integral lattice $L$, and $q_{L}$ the associated discriminant quadratic form [29], i.e., the form

$$
q_{L}: A_{L} \rightarrow \mathbb{Q} / 2 \mathbb{Z}
$$

induced by the quadratic form on $L$. More generally, we denote by $A_{q}$ a finite abelian group with a quadratic form $q: A_{q} \rightarrow \mathbb{Q} / 2 \mathbb{Z}$. The quadratic form $q$ determines a bilinear form on $A_{q}$ which takes values in $\mathbb{Q} / \mathbb{Z}$; we denote it by $a \cdot b$, where $a, b \in A_{q}$.

A sublattice $L^{\prime}$ of a lattice $L$ is called primitive if $L / L^{\prime}$ is a free group; in other words, $L^{\prime}=\left(L^{\prime} \otimes \mathbb{Q}\right) \cap L$. Correspondingly, a primitive embedding of a lattice $L^{\prime}$ in $L$ is an embedding such that the image is primitive.

If $L$ is a primitive sublattice of a unimodular lattice $\Gamma$ and $L^{\perp}$ is its orthogonal complement in $\Gamma$, then there is an isomorphism between the discriminant groups [29] 


$$
\gamma: A_{L} \stackrel{\cong}{\rightrightarrows} A_{L^{\perp}}
$$

that flips the sign of the quadratic form

$$
q_{L}=-q_{L^{\perp}} \circ \gamma
$$

More precisely, $\bar{x} \cong \bar{y}$, with $\bar{x} \in A_{L}$ and $\bar{y} \in A_{L^{\perp}}$, if and only if $x+y \in \Gamma$ for any choice $x \in L^{*}, y \in\left(L^{\perp}\right)^{*}$ of representatives of $\bar{x}, \bar{y}$. Conversely, given two even lattices $L_{1}, L_{2}$ with isomorphic discriminant groups $\gamma: A_{L_{1}} \stackrel{\cong}{\rightrightarrows} A_{L_{2}}$ and opposite discriminant quadratic forms $q_{L_{1}}=-q_{L_{2}} \circ \gamma$, one can construct an even unimodular lattice $\Gamma$ by "gluing" $L_{1}$ and $L_{2}$, i.e.,

$$
\Gamma=\left\{x \oplus y \in L_{1}^{*} \oplus L_{2}^{*} \mid \bar{x} \cong \bar{y}\right\}
$$

where $\bar{x}, \bar{y}$ are the images in $A_{L_{1}}, A_{L_{2}}$ of $x \in L_{1}^{*}$ and $y \in L_{2}^{*}$, respectively.

\section{Appendix A.3. The Leech lattice and the Golay code}

The Leech lattice can be defined in terms of the (extended) binary Golay code $\mathcal{C}_{24}$, a 12 -dimensional subspace of the vector space $\mathbb{F}_{2}^{24}$, where $\mathbb{F}_{2}=$ $\{0,1\}$ is the field with two elements. To each vector $f=\left(f_{1}, \ldots, f_{24}\right)$ of $\mathcal{C}_{24}$ (i.e., to each codeword) we associate a subset $X_{f}$ of $\Omega=\{1, \ldots, 24\}$, corresponding to the non-zero coordinates of $f$, i.e., $X_{f}=\left\{i \in \Omega \mid f_{i} \neq 0\right\}$. This collection of $2^{12}=4096$ subsets of $\Omega$ (called $\mathcal{C}$-sets) includes the empty set, $\Omega$ itself, $759 \mathcal{C}$-sets with eight elements (special octads), 2576 with 12 elements and 759 with 16 elements (the complements in $\Omega$ of the special octads). Furthermore, for any choice of five distinct elements in $\Omega$ there is a unique special octad containing them. These properties are sufficient to determine the collection of $\mathcal{C}$-sets, and thus the (extended) Golay code, up to permutations of the objects in $\Omega$ (see [19], Chap. 10.2.1). The Mathieu group $\mathbb{M}_{24}$ can be defined as the group of automorphisms of the (extended) Golay code, or, equivalently, as the subgroup of $S_{24}$ stabilizing the collection of $\mathcal{C}$-sets.

An explicit description of the Leech lattice $\Lambda \subset \mathbb{R}^{24}$ can be given as follows (see [19], Chap. 10.3): the vector $v=\frac{1}{\sqrt{8}}\left(v_{1}, \ldots, v_{24}\right)$ is an element of the Leech lattice provided that

- the $v_{i}, i=1, \ldots, 24$, are all integers of the same parity;

$-\sum_{i=1}^{24} v_{i} \equiv 0$ or $4 \bmod 8$ according to $v_{i} \equiv 0$ or $1 \bmod 2$, respectively; and

- for each $\nu \in\{0,1,2,3\}$, the set $\left\{i \in \Omega \mid v_{i} \equiv \nu \bmod 4\right\}$ is a $\mathcal{C}$-set. 


\section{Appendix B. Proof of the Theorem}

In this appendix, we give the remaining details of the arguments of Section 2, leading to the proof of the Theorem.

\section{Appendix B.1. $G$ as a subgroup of $O\left(\Gamma^{25,1}\right)$}

For each lattice $L$ and group $G \subset \operatorname{Aut}(L)$, we define as in (2.4) the $G$-invariant lattice $L^{G}$ as $L^{G}=\{x \in L \mid g(x)=x, \forall g \in G\}$. Furthermore, $L_{G}$ is its orthogonal complement in $L, L_{G}=\left\{x \in L \mid x \cdot y=0, \forall y \in L^{G}\right\}$. We take $L=\Gamma^{4,20} \subset \mathbb{R}^{4,20}$, and consider the case where $G$ is the subgroup of $\operatorname{Aut}\left(\Gamma^{4,20}\right) \subset O(4,20, \mathbb{R})$, fixing a positive-definite four plane $\Pi \subset \mathbb{R}^{4,20}$.

Proposition B.1. For any choice of a positive four-plane $\Pi, L_{G}$ is a negative definite lattice of rank $\operatorname{rk} L_{G} \leq 20$. G acts trivially on $A_{L_{G}}$, and $l\left(L_{G}\right) \leq 24-\operatorname{rk}\left(L_{G}\right)$.

Proof. The first part is obvious. By definition, $G$ acts trivially on $L^{G}$, and hence its induced action on $A_{L^{G}}$ is also trivial. Since $L^{G}$ and $L_{G}$ are orthogonal primitive sublattices of the self-dual lattice $L$, it follows that for each $y \in\left(L_{G}\right)^{*}$ there exists a vector $v=x+y \in L$ with $x \in\left(L^{G}\right)^{*}$. For all $w \in L^{G}$ and any lattice automorphism $g \in G, w \cdot g(v)=g(w) \cdot g(v)=w \cdot v$, so that $g(v)-v \in\left(L^{G}\right)^{\perp}=L_{G}$. Since $g$ is linear and fixes $x \in\left(L^{G}\right)^{*}$, we have $g(v)-$ $v=g(x+y)-(x+y)=g(y)-y$. It follows that $g(y) \equiv y \bmod L_{G}$, so that $G$ acts trivially on $A_{L_{G}}=\left(L_{G}\right)^{*} / L_{G}$. Finally, $l\left(L_{G}\right)=l\left(L^{G}\right) \leq \operatorname{rk}\left(L^{G}\right)$, and the last statement follows.

Up to isomorphism, there is a unique even unimodular lattice $\Gamma^{25,1}$ of signature $(25,1)$. The lattice $\Gamma^{25,1}$ can be defined as the (additive) subgroup of $\mathbb{R}^{25,1}$ with elements $\left(x_{0}, \ldots, x_{24} ; x_{25}\right)$ such that

$$
x_{0}+\cdots+x_{24}-x_{25} \in 2 \mathbb{Z}
$$

where either $x_{i} \in \mathbb{Z}$ for all $i$, or $x_{i} \in \mathbb{Z}+\frac{1}{2}$ for all $i$ (see [19], Chap. 26). In the rest of this subsection, we will prove that $L_{G}(-1)$ can be embedded into $\Gamma^{25,1}$.

Recall that, for any prime $p$, a $p$-group is a group whose order is a power of $p$. A Sylow $p$-subgroup of a group $G$ is a maximal $p$-subgroup, i.e., a $p$-subgroup of $G$ which is not a proper subgroup of any other $p$-subgroup. For abelian groups, there is a unique Sylow $p$-subgroup for each prime $p$, the subgroup of elements whose order is a power of $p$. For more general 
finite groups, for each given $p$ the Sylow $p$-subgroups are all isomorphic and related by conjugation.

Let $A_{q}$ be a finite abelian group with quadratic form $q: A_{q} \rightarrow \mathbb{Q} / 2 \mathbb{Z}$. For any prime $p$, let $A_{q_{p}}$ be the Sylow $p$-subgroup of $A_{q}$, and $q_{p}$ the restriction of $q$ to $A_{q_{p}}$. Note that if $a \in A_{q_{p}}$, then for any $b \in A_{q}$

$$
p^{n}(a \cdot b) \equiv 0 \quad \bmod \mathbb{Z},
$$

where $p^{n}$ is the order of $a$, and $a \cdot b$ is the bilinear form induced by $q$. In particular, if $b \in A_{q_{p^{\prime}}}$, with $p^{\prime} \neq p$, this implies $a \cdot b \equiv 0 \bmod \mathbb{Z}$. Thus, we have an orthogonal decomposition of the quadratic form $q=\oplus_{p} q_{p}$, where each $q_{p}$ is a quadratic form on an abelian $p$-group.

It follows from Theorem 1.12.2 of [29] that an even lattice $L$ of signature $\left(t^{+}, t^{-}\right)$and discriminant group $A_{q}$ can be primitively embedded into some even unimodular lattice of signature $\left(d^{+}, d^{-}\right)$, provided that

$$
\begin{aligned}
& d^{+}-d^{-} \equiv 0 \bmod 8 \\
& d^{-}-t^{-} \geq 0, \quad d^{+}-t^{+} \geq 0, \quad d^{+}+d^{-}-t^{-}-t^{+} \geq l\left(A_{q}\right), \\
& d^{-}+d^{+}-t^{-}-t^{+}>l\left(A_{q_{p}}\right), \quad \text { for all odd primes } p, \\
& \text { (B.3d) } d^{-}+d^{+}-t^{-}-t^{+}>l\left(A_{q_{2}}\right) \quad \text { or } \quad q_{2}=q_{\theta}^{(2)}(2) \oplus q_{2}^{\prime} \text {, for some } q_{2}^{\prime} \text {, }
\end{aligned}
$$

where $q_{\theta}^{(2)}(2)$ is the discriminant quadratic form of the $\mathfrak{s u}(2)$ root lattice $A_{1} \cong \mathbb{Z}(2)$.

Proposition B.2. The lattice $L_{G}(-1)$ can be primitively embedded into $\Gamma^{25,1}$. The action of $G$ can be extended to an action on $\Gamma^{25,1}$ such that the $G$-invariant sublattice $\left(\Gamma^{25,1}\right)^{G}$ is the orthogonal complement of $L_{G}(-1)$ in $\Gamma^{25,1}$, and such that $\left(\Gamma^{25,1}\right)^{G}$ contains an element of norm 2.

Proof. Let us prove that we can embed $L_{G}(-1) \oplus A_{1}$ into $\Gamma^{25,1}$, where $A_{1}$ denotes the root lattice of the $\mathfrak{s u}(2)$ Lie algebra. Condition (B.3a) obviously holds. By Proposition B.1, we have $\operatorname{rk}\left(L_{G}(-1) \oplus A_{1}\right)+l\left(L_{G}(-1) \oplus\right.$ $\left.A_{1}\right) \leq 26$, so that also (B.3b) is satisfied. Let $A_{q}$ be the discriminant group of $L_{G}(-1) \oplus A_{1}$, with discriminant quadratic form $q$. Let us consider the decomposition $q=\oplus_{p} q_{p}$ where, for each prime $p, q_{p}$ is the restriction of $q$ to the $p$-Sylow subgroup $A_{q_{p}}$. Since the discriminant group of $A_{1}$ is $\mathbb{Z} / 2 \mathbb{Z}$, we have $l\left(A_{q_{p}}\right) \leq l\left(L_{G}\right)<26-\operatorname{rk}\left(L_{G} \oplus A_{1}\right)$ for all odd $p$, and (B.3c) holds. Finally, it is clear that $q_{2}=q_{\theta}^{(2)}(2) \oplus q_{2}^{\prime}$, where $q_{\theta}^{(2)}(2)$ is the discriminant form of $A_{1}$ and $q_{2}^{\prime}$ is the restriction of $q_{L_{G}}$ to the 2-Sylow subgroup. Thus, 
it follows from (B.3) that $L_{G}(-1) \oplus A_{1}$ can be primitively embedded into $\Gamma^{25,1}$.

Since $G$ acts trivially on $A_{L_{G}}$, the action of $G$ on $L_{G}(-1)$ can be extended to an action on $\Gamma^{25,1}$ which acts trivially on the orthogonal complement of $L_{G}(-1)$ in $\Gamma^{25,1}$. Thus, $\left(\Gamma^{25,1}\right)^{G} \cong\left(L_{G}(-1)\right)^{\perp}$, and $A_{1}$ is a sublattice of $\left(\Gamma^{25,1}\right)^{G}$, so that $\left(\Gamma^{25,1}\right)^{G}$ contains a vector of norm 2 .

\section{Appendix B.2. $G$ as a subgroup of $\mathrm{Co}_{0}$}

The automorphism group $\operatorname{Aut}\left(\Gamma^{25,1}\right)$ of $\Gamma^{25,1}$ is generated by the sign flip $x_{i} \mapsto-x_{i}$, together with the subgroup of autochronous transformations $\mathrm{Aut}^{+}\left(\Gamma^{25,1}\right)$ which stabilize the cone of positive time vectors in $\mathbb{R}^{25,1}$ (see [19], Chap. 27). The group $\mathrm{Aut}^{+}\left(\Gamma^{25,1}\right)$ contains a normal subgroup $W$ (the Weyl group), generated by the reflections $R_{r}$ with respect to the hyperplanes $r^{\perp}$

$$
R_{r}(x)=x-(x \cdot r) r, \quad x \in \Gamma^{25,1}
$$

where $r$ is any root in $r \in \Gamma^{25,1}$, i.e., satisfies $r \cdot r=2$. The complement in $\mathbb{R}^{25,1}$ of the union $\bigcup_{r \cdot r=2} r^{\perp}$ of the corresponding hyperplanes has infinitely many connected components, and the closure of each component is called a Weyl chamber.

The group of autochronous transformations is the semidirect product $W \rtimes \mathrm{Co}_{\infty}$, where $\mathrm{Co}_{\infty}$ is the automorphism group of the Dynkin diagram of $W$ (see [19], Chap. 27). The groups $W$ and $\mathrm{Co}_{\infty}$ can be described more explicitly upon choosing a set of generators for $W$, i.e., a set of fundamental roots. One convenient choice is given by the set of Leech roots, i.e., by the vectors $r \in \Gamma^{25,1}$ with

$$
r \cdot r=2 \quad r \cdot w=-1
$$

where $w$ is the null (Weyl) vector

$$
w=(0,1,2,3, \ldots, 23,24 ; 70) \in \Gamma^{25,1} .
$$

The sublattice $\Gamma^{25,1} \cap w^{\perp}$ is degenerate, while its quotient $\left(\Gamma^{25,1} \cap w^{\perp}\right) / w$ is the Leech lattice $\Lambda$ (see [19], Chap. 26), the unique positive even unimodular lattice of rank 24 containing no roots. The automorphism group $\mathrm{Co}_{\infty}$ of the Dynkin diagram of $W$ contains $\operatorname{Aut}(\Lambda)=\mathrm{Co}_{0}$, the automorphism group of the Leech lattice, as the subgroup which fixes a given reference Leech root $\bar{r}$. 
The group $\mathrm{Co}_{\infty}$ is generated by $\mathrm{Co}_{0}$, together with the translations of the Leech roots by vectors in $\Lambda$.

Let us consider the embedding of $L_{G}(-1) \oplus A_{1}$ into $\Gamma^{25,1}$. Clearly, the sign flip does not fix any sublattice of $\Gamma^{25,1}$, so that $G \subseteq \operatorname{Aut}^{+}\left(\Gamma^{25,1}\right)$ (note that the sign flip is central in $\left.\operatorname{Aut}\left(\Gamma^{25,1}\right)\right)$. Note that the lattice $\left(\Gamma^{25,1}\right)^{G}$ always contains a vector in the interior of some Weyl chamber. For if this was not true, then $\left(\Gamma^{25,1}\right)^{G}$ would be contained in one of the hyperplanes orthogonal to some root $r$, and thus $r \in\left(\Gamma^{25,1}\right)_{G}=L_{G}(-1)$. But this would contradict our assumption that $L_{G}$ contains no vector of norm -2 . Since $W$ acts transitively on the Weyl chambers, we can choose our embedding of $L_{G}(-1)$ into $\Gamma^{25,1}$ such that $\left(\Gamma^{25,1}\right)^{G}$ contains a vector in the interior $K$ of the fundamental Weyl chamber containing $w$. Since $K \cap t(K)=\emptyset$, for all non-trivial $t \in W$, it follows that $G$ must be contained in $\mathrm{Co}_{\infty}$. Since $w$ is fixed by $\mathrm{Co}_{\infty}$, we have $\left(\Gamma^{25,1}\right)_{G} \subset\left(\Gamma^{25,1} \cap w^{\perp}\right)$, and the projection $\left(\Gamma^{25,1} \cap\right.$ $\left.w^{\perp}\right) \rightarrow\left(\Gamma^{25,1} \cap w^{\perp}\right) / w \cong \Lambda$ induces an embedding of $\left(\Gamma^{25,1}\right)_{G}$ into the Leech lattice $\Lambda$

$$
L_{G}(-1) \cong\left(\Gamma^{25,1}\right)_{G} \subset\left(\Gamma^{25,1} \cap w^{\perp}\right) \rightarrow\left(\Gamma^{25,1} \cap w^{\perp}\right) / w \cong \Lambda
$$

As in Proposition B.2, the action of $G$ on $\left(\Gamma^{25,1}\right)_{G}$ can be extended to an action on $\Lambda$, such that $L_{G}(-1) \cong\left(\Gamma^{25,1}\right)_{G} \cong \Lambda_{G}$ is the orthogonal complement of the sublattice $\Lambda^{G} \subset \Lambda$ fixed by $G$. We conclude that $G$ is a subgroup of $\operatorname{Aut}(\Lambda) \cong \mathrm{Co}_{0}$ fixing a sublattice $\Lambda^{G}$ of rank at least 4 , thus proving the proposition in Section 2.

\section{Appendix B.3. The proof of the Theorem}

The stabilizers of sublattices of the Leech lattice have been classified [20,26]. We will use this classification to prove now the Theorem stated in the Introduction. The action of $\operatorname{Aut}(\Lambda) \cong \mathrm{Co}_{0}$ is well defined on the classes of the quotient $\Lambda / 2 \Lambda$, because $2 \Lambda$ is stable under lattice automorphisms. In particular, if $v \in \Lambda$ is fixed by the action of $G$, then $G$ must be in the stabilizer of the class of $\Lambda / 2 \Lambda$ containing $v$. Note that opposite vectors $x,-x \in \Lambda$ are contained in the same class in this quotient. More generally, if we define the short vectors $x \in \Lambda$ to be the vectors of norm $x^{2} \leq 8$, then for each nontrivial class in $\Lambda / 2 \Lambda$, one of the following mutually exclusive alternatives holds [19]:

(1) the class contains exactly one pair of short vectors $\pm x \in \Lambda$ of type 2 $\left(x^{2}=4\right)$; 
(2) the class contains exactly one pair of short vectors $\pm x \in \Lambda$ of type 3 $\left(x^{2}=6\right)$;

(3) the class contains exactly 24 pairs of short vectors $\pm x_{1}, \ldots, \pm x_{24} \in \Lambda$ of type 4 , that are mutually orthogonal $\left(x_{i} \cdot x_{j}=8 \delta_{i j}\right)$.

Thus, each primitive vector $v \in \Lambda^{G}$ is congruent modulo $2 \Lambda$ to some short vector $v_{\mathrm{s}} \in \Lambda, v_{\mathrm{s}}^{2} \leq 8$. We can now distinguish the following cases:

Case 1: Suppose there is a vector $v \in \Lambda^{G}$ which is congruent modulo $2 \Lambda$ to a short vector of norm 8 . Then the class of $v$ in $\Lambda / 2 \Lambda$ contains 24 mutually orthogonal pairs $\pm x_{1}, \ldots, \pm x_{24} \in \Lambda$ of norm 8 . The subgroup of $\mathrm{Co}_{0}$ stabilizing such a class is the semidirect product $\mathbb{Z}_{2}^{12} \rtimes \mathbb{M}_{24}$ (see [20] and Chap. 10 of [19], ). Here, $\mathbb{M}_{24}$ acts by permutations of the 24 pairs $\pm x_{1}, \ldots, \pm x_{24}$, while each element $\epsilon_{f} \in \mathbb{Z}_{2}^{12}$ is associated to a codeword $f \equiv\left(f_{1}, \ldots, f_{24}\right) \in(\mathbb{Z} / 2 \mathbb{Z})^{24}$ in the (extended) binary Golay code ( $[19]$, chapter 11) and acts by

$$
\epsilon_{f}\left(x_{i}\right)=(-1)^{f_{i}} x_{i}, \quad i=1, \ldots, 24,
$$

on the vectors of the class. Thus, $G$ is a subgroup of $\mathbb{Z}_{2}^{12} \rtimes \mathbb{M}_{24}$ that fixes a subspace of dimension at least 4 . This realizes case (i) of the Theorem. In particular, $G^{\prime \prime} \subset \mathbb{M}_{24}$ can be any subgroup with at least four orbits $\Omega_{1}, \ldots, \Omega_{4}$ when acting on $\{1, \ldots, 24\}$, and $G^{\prime}$ is generated by the $\epsilon_{f} \in \mathbb{Z}_{2}^{12}$ such that $f_{i}=0$ for all $i \in \Omega_{1} \sqcup \ldots \sqcup \Omega_{4}$.

Case 2: If Case 1 does not apply, then each primitive vector $v \in \Lambda^{G}$ is congruent modulo $2 \Lambda$ to a pair of short vectors $\pm v_{\mathrm{s}} \in \Lambda$ with $v_{\mathrm{s}}^{2} \leq 6$. Let us assume that, for each $v \in \Lambda^{G}$, the corresponding short vector $v_{\mathrm{s}}$ is also contained in $\Lambda^{G}$ (the situations where this assumption does not hold are considered in Case 3 below). Since $\Lambda^{G}$ is primitive, $\left(v-v_{\mathrm{s}}\right) / 2 \in \Lambda$ is also contained in $\Lambda^{G}$. The sublattices $S \subset \Lambda$ containing only short vectors of norm 4 and 6 and such that each primitive vector is congruent to a short one $\bmod 2 S$ are called $\mathcal{S}$-lattices, and they have been completely classified [26]. In particular, up to automorphisms, there are only three $\mathcal{S}$-lattices of rank at least $4[20]$ :

$\begin{array}{cccc}S & \text { rk } S & \operatorname{Stab}(S) & \operatorname{Aut}(S) \\ 2^{9} 3^{6} & 4 & \mathbb{Z}_{3}^{4} \rtimes A_{6} & \mathbb{Z}_{2} \times\left(S_{3} \times S_{3}\right) \cdot \mathbb{Z}_{2} \\ 2^{5} 3^{10} & 4 & 5^{1+2} \cdot \mathbb{Z}_{4} & \mathbb{Z}_{2} \times S_{5} \\ 2^{27} 3^{36} & 6 & 3^{1+4} \cdot \mathbb{Z}_{2} & \mathbb{Z}_{2} \times U_{4}(2) \cdot \mathbb{Z}_{2} .\end{array}$


Since $\Lambda^{G}$ is a $\mathcal{S}$-lattice of rank at least $4, G$ must be a subgroup of one of the groups $\operatorname{Stab}(S)$ from above, corresponding to the cases (ii), (iii) and (iv) (with $G^{\prime \prime}$ trivial) of the Theorem.

Case 3: The last case arises if each primitive vector $v \in \Lambda^{G}$ is congruent modulo $2 \Lambda$ to a pair of short vectors $\pm v_{\mathrm{s}} \in \Lambda$ with $v_{\mathrm{s}}^{2} \leq 6$, but some of these short vectors are not contained in $\Lambda^{G}$. In this case, we define a finite chain of sublattices of $\Lambda$

$$
\Lambda^{G}=S_{0} \subset S_{1} \subset \cdots \subset S_{N}=S
$$

where each $S_{i+1}$ is defined in terms of $S_{i}$ as follows [30]:

(1) If $S_{i}$ is contained in an $\mathcal{S}$-lattice of the same rank or if $S_{i}$ contains a vector congruent modulo $2 \Lambda$ to a short vector of norm 8 , then we set $S=S_{i}$ and the procedure stops.

(2) Otherwise, if $S_{i}$ contains a vector $v \in 2 \Lambda$ with $v / 2 \notin S_{i}$, then $S_{i+1}$ is obtained by adjoining $v / 2$ to $S_{i}$. There are only a finite number of vectors to check, namely one representative for each class in $S_{i} / 2 S_{i}$. Note that $S_{i} \otimes \mathbb{Q}=S_{i+1} \otimes \mathbb{Q}$, so that $\operatorname{rk} S_{i}=\operatorname{rk} S_{i+1}$ and $\operatorname{Stab}\left(S_{i}\right)=$ $\operatorname{Stab}\left(S_{i+1}\right)$.

If neither of these cases applies, then there is a non-empty set of short vectors $v_{\mathrm{s}} \notin S_{i} \otimes \mathbb{Q}$, with $0<v_{\mathrm{s}}^{2} \leq 6$, congruent modulo $2 \Lambda$ to some $v \in S_{i}$. Then $S_{i+1}$ is defined as follows:

(3) If one of the short vectors is such that $v_{\mathrm{s}} \cdot w \neq 0$ for some $w \in S_{i}$, then $S_{i+1}$ is obtained by adjoining $v_{\mathrm{s}}$ to $S_{i}$. Any element $g \in \operatorname{Stab}\left(S_{i}\right)$ must preserve the class of $v_{\mathrm{s}}$ in $\Lambda / 2 \Lambda$, so that $g\left(v_{\mathrm{s}}\right) \in\left\{ \pm v_{\mathrm{s}}\right\}$, and also the product $v_{\mathrm{s}} \cdot w \neq 0$. Thus, the only possibility is $g\left(v_{\mathrm{s}}\right)=v_{\mathrm{s}}$, so that $\operatorname{Stab}\left(S_{i+1}\right)=\operatorname{Stab}\left(S_{i}\right)$. Furthermore, we have a strict inequality $\operatorname{rk} S_{i+1}>\operatorname{rk} S_{i}$.

(4) If all the short vectors $v_{\mathrm{s}}$ are orthogonal to $S_{i}$, we choose one of them and define $S_{i+1}$ as the $\mathbb{Z}$-linear span of $S_{i}$ and $v_{\mathrm{s}}$. The only nontrivial action of an element $g \in \operatorname{Stab}\left(S_{i}\right)$ on $S_{i+1}$ is $g\left(v_{\mathrm{s}}\right)=-v_{\mathrm{s}}$, so that $\left|\operatorname{Stab}\left(S_{i}\right): \operatorname{Stab}\left(S_{i+1}\right)\right| \leq 2$. Furthermore, $\operatorname{rk} S_{i+1}>\operatorname{rk} S_{i}$.

The stabilizer $\operatorname{Stab}(S)$ of the lattice $S$ at the end of the chain must be a subgroup of $\mathbb{Z}_{2}^{12} \rtimes \mathbb{M}_{24}$ or one of the stabilizers of the $\mathcal{S}$-lattices above. However, if the case (4) of the above procedure occurs for some intermediate $S_{i}, \operatorname{Stab}(S)$ might be just a normal subgroup of $G$. Our analysis is greatly 
simplified by the following result, which is an immediate consequence of Lemma 4.8 of [30].

Proposition B.3. If $\mathrm{rk} S_{i}>3$ and none of the cases (1), (2) and (3) applies, then $S_{i}$ is a sublattice of the $\mathcal{S}$-lattice $2^{27} 3^{36}$.

It is easy to see that if $S_{i}$ is a sublattice of an $\mathcal{S}$-lattice also $S_{i+1}$ is. Since the starting point $S_{0}=\Lambda^{G}$ of the chain has already rank greater than 3 , this proposition implies that if $\Lambda^{G}$ is not a sublattice of $2^{27} 3^{36}$, then case (4) above never occurs. Thus, in this case, $G \cong \operatorname{Stab}(S)$ and we reobtain the groups (i), (ii) or (iii) of the Theorem. If $\Lambda^{G}$ is a sublattice of $2^{27} 3^{36}$, then we have an inverse chain of inclusions for the stabilizers

$$
\operatorname{Stab}(S) \subseteq \cdots \subseteq \operatorname{Stab}\left(S_{1}\right) \subseteq \operatorname{Stab}\left(S_{0}\right)=G
$$

where each stabilizer group is a subgroup of index at most 2 in the previous one

$$
\left|\operatorname{Stab}\left(S_{i}\right): \operatorname{Stab}\left(S_{i+1}\right)\right| \leq 2
$$

Furthermore, whenever $\left|\operatorname{Stab}\left(S_{i}\right): \operatorname{Stab}\left(S_{i+1}\right)\right|=2$, we have $\operatorname{rk} S_{i+1}>\operatorname{rk} S_{i}$, and since $\operatorname{rk} S_{2^{27} 3^{36}}-\operatorname{rk} \Lambda^{G} \leq 2$, we have

$$
\frac{|G|}{\left|\operatorname{Stab}\left(S_{2^{27} 3^{36}}\right)\right|} \leq 2^{2} \text {. }
$$

Thus, $G$ is the extension of $\operatorname{Stab}\left(S_{2^{27} 3^{36}}\right)$ by a group $G^{\prime \prime}$ of order at most 4 , and all the possibilities are considered in the case (iv) of the Theorem. This completes the proof of the Theorem.

\section{Appendix B.4. Realizing all symmetry groups of K3}

Let $G \subset \operatorname{Aut}(\Lambda)$ be the pointwise stabilizer of a sublattice $S \cong \Lambda^{G}$ of rank $\operatorname{rk} S \geq 4$. In this section, we will prove that, for each such $G$, there exists a non-linear $\sigma$-model on K3 having $G$ as its group of symmetries. This result is based on the assumption that for every choice of a positive definite fourdimensional subspace $\Pi \subset \mathbb{R}^{4,20}$ such that no root $v \in \Gamma^{4,20}$ is orthogonal to $\Pi$, the corresponding non-linear $\sigma$-model is well defined.

Let $d+4$, with $d \geq 0$, be the rank of $S$ and let $S^{\perp} \equiv \Lambda_{G}$ be its orthogonal complement in $\Lambda$. Suppose that $S^{\perp}(-1)$, obtained from $S^{\perp}$ by changing the sign of its quadratic form, can be primitively embedded into $\Gamma^{4,20}$. Then, by a reasoning similar to Proposition B.2, we conclude that the action of $G$ on $S^{\perp}(-1)$ can be extended to $\Gamma^{4,20}$, in such a way that $\left(\Gamma^{4,20}\right)_{G} \cong S^{\perp}(-1)$, i.e., 
that the sublattice $\left(\Gamma^{4,20}\right)^{G}$ invariant under $G$ is the orthogonal complement of $S^{\perp}(-1)$. Since $\left(\Gamma^{4,20}\right)^{G}$ has signature $(4, d)$, one can always find a positive definite four-dimensional subspace $\Pi \subset \mathbb{R}^{4,20}$ such that $\left(\Gamma^{4,20}\right)_{G}=\Gamma^{4,20} \cap \Pi^{\perp}$. Furthermore, $\left(\Gamma^{4,20}\right)_{G}$ contains no vectors of norm $-2\left(\right.$ because $\left(\Gamma^{4,20}\right)_{G}(-1) \cong$ $S^{\perp} \subset \Lambda$ and $\Lambda$ contains no vectors of norm 2$)$, and $G$ is the subgroup of $O\left(\Gamma^{4,20}\right)$ fixing $\Pi$ pointwise. Following the arguments of Section $2, \Pi$ corresponds to a well-defined non-linear $\sigma$-model with symmetry group $G$.

Thus it remains to prove that a primitive embedding of $S^{\perp}(-1)$ into $\Gamma^{4,20}$ always exists. The "gluing" construction described in Appendix B.1 shows that, for an even lattice with signature $\left(t_{+}, t_{-}\right)$and discriminant form $q-$ we will denote them as a triple $\left(t_{+}, t_{-} ; q\right)$ — the existence of an embedding into some even unimodular lattice with signature $\left(l_{+}, l_{-}\right)$is equivalent to the existence of an even lattice with signature and discriminant form $\left(l_{+}-\right.$ $\left.t_{+}, l_{-}-t_{-},-q\right)$. In [29, Theorem 1.12.4], the following sufficient conditions are proved for the existence of such an embedding:

(1) $l_{+}-l_{-} \equiv 0 \bmod 8$

(2) $t_{+} \leq l_{+}, t_{-} \leq l_{-}$and $t_{+}+t_{-} \leq \frac{1}{2}\left(l_{+}+l_{-}\right)$.

The lattice $S$ has signature $(4+d, 0)$, so that it can be embedded into an even unimodular lattice $\Gamma^{8+d, d} \cong E_{8} \oplus U^{d}$ of signature $(8+d, d)$, where $U$ is the unimodular lattice of signature $(1,1)$. Let $S^{\prime}$ be the orthogonal complement of $S$ in $\Gamma^{8+d, d}$. If $q$ denotes the discriminant form of $S$, then $S^{\prime}, S^{\perp}$ and $S^{\perp}(-1)$ have signature and discriminant form $(4, d ;-q),(20-d, 0 ;-q)$ and $(0,20-d ; q)$, respectively. By comparing the signatures and discriminant forms of $S^{\perp}(-1)$ and $S^{\prime}$, we conclude that these two lattices can be "glued" together to form the even unimodular lattice $\Gamma^{4,20}$.

\section{Appendix C. Gepner models}

Here we collect, following Brunner and Gaberdiel [31], our conventions for the description of $\mathcal{N}=2$ minimal models and Gepner models.

\section{Appendix C.1. $\mathcal{N}=2$ Minimal models and Gepner models at $c=6$}

The $\mathcal{N}=2$ minimal model at level $k$ has central charge $c=\frac{3 k}{k+2}$, and can be described in terms of the coset

$$
\frac{\mathfrak{s u}(2)_{k+2} \oplus \mathfrak{u}(1)_{4}}{\mathfrak{u}(1)_{2 k+4}}
$$


that captures the bosonic subalgebra of the $\mathcal{N}=2$ superconformal algebra. The coset representations are labelled by

$$
(l, m, s), \quad l=0, \ldots, k, \quad m \in \mathbb{Z}_{2 k+4}, \quad s \in \mathbb{Z}_{4},
$$

subject to the condition

$$
l+m+s=0 \quad \bmod 2
$$

and with the field identification

$$
(l, m, s) \sim(k-l, m+k+2, s+2) .
$$

We denote by $[l, m, s]$ the class corresponding to $(l, m, s)$. In terms of the $\mathcal{N}=2$ algebra, the irreducible representations are of the form $\mathcal{H}_{(l, m, s)} \oplus$ $\mathcal{H}_{(l, m, s+2)}$ with $s$ even (odd) for the NS (R) sector, since the fermionic generators of the $\mathcal{N}=2$ algebra map $\mathcal{H}_{(l, m, s)}$ to $\mathcal{H}_{(l, m, s+2)}$. The conformal weight and $U(1)$-charge of the ground state in the $(l, m, s)$ sector are given by

$$
h_{l, m, s}=\frac{l(l+2)-m^{2}}{4(k+2)}+\frac{s^{2}}{8} \quad \bmod \mathbb{Z}, \quad q_{l, m, s}=\frac{m}{k+2}-\frac{s}{2} \quad \bmod 2 \mathbb{Z} .
$$

The character of the $(l, m, s)$ coset representation equals (with $q=\mathrm{e}^{2 \pi \mathrm{i} \tau}$, $\left.y=\mathrm{e}^{2 \pi \mathrm{i} z}\right)$

$$
\begin{aligned}
\chi_{[l, m, s]}(\tau, z) & =\operatorname{Tr}_{\mathcal{H}_{[l, m, s]}}\left(q^{L_{0}-\frac{c}{24}} y^{J_{0}}\right) \\
& =\sum_{j \in \mathbb{Z}} c_{m+4 j-s}^{l}(\tau) q^{\frac{k+2}{2 k}\left(\frac{m}{k+2}-\frac{s}{2}+2 j\right)^{2}} y^{\frac{m}{k+2}-\frac{s}{2}+2 j}
\end{aligned}
$$

Here, $c_{m}^{l}(\tau)$ can be obtained from the identity

$$
\sum_{m \in \mathbb{Z} / 2 k \mathbb{Z}} c_{m}^{l}(\tau) \theta_{m, k}(\tau, z)=\frac{\theta_{l+1, k+2}(\tau, z)-\theta_{-l-1, k+2}(\tau, z)}{\theta_{1, k+2}(\tau, z)-\theta_{-1, k+2}(\tau, z)},
$$

where $\theta_{m, k}(\tau, z)$ is the $\mathfrak{s u}(2)$ theta function

$$
\theta_{m, k}(\tau, z)=\sum_{n \in \mathbb{Z}} q^{k\left(n+\frac{m}{2 k}\right)^{2}} y^{k\left(n+\frac{m}{2 k}\right)}
$$


with $m \in \mathbb{Z} / 2 k \mathbb{Z}$. For the calculation of the elliptic genus we are interested in the trace with the insertion of $(-1)^{F}$; for the $\mathcal{N}=2$ representation corresponding to $(l, m, s)$, this leads to

$$
I_{m}^{l}(\tau, z)=\chi_{[l, m, s]}(\tau, z)-\chi_{[l, m, s+2]}(\tau, z),
$$

where $s=0$ in the NS sector and $s=1$ in the Ramond sector.

A Gepner model at $c=6$ is defined as a $\mathbb{Z}_{H}$ orbifold of a tensor product $\left(k_{1}\right) \cdots\left(k_{r}\right)$ of $\mathcal{N}=2$ minimal models, where

$$
\sum_{i=1}^{r} \frac{3 k_{i}}{k_{i}+2}=6, \quad \text { and } \quad H=\operatorname{lcm}\left\{k_{i}+2\right\}
$$

The spectrum of the orbifold theory is given by

$$
\bigoplus_{\substack{n \in \mathbb{Z}_{H} \\\left[l_{i}, m_{i}, s_{i}\right]}} \bigotimes_{i=1}^{r} \mathcal{H}_{\left[l_{i}, m_{i}+n, s_{i}\right]} \otimes \overline{\mathcal{H}}_{\left[l_{i}, m_{i}-n, \bar{s}_{i}\right]}
$$

and the sum is over the sectors $\left[l_{i}, m_{i}, s_{i}\right]$ satisfying the orbifold conditions

$$
\begin{aligned}
& \sum_{i=1}^{r} \frac{m_{i}}{k_{i}+2} \in \mathbb{Z}, \quad r \text { even, } \\
& \sum_{i=1}^{r} \frac{m_{i}}{k_{i}+2}-\frac{s_{1}}{2} \in \mathbb{Z}, \quad r \text { odd }
\end{aligned}
$$

together with the spin alignment condition

$$
s_{i}-s_{j} \in 2 \mathbb{Z}, \quad i, j=1, \ldots, r .
$$

We denote a state transforming in the $\mathcal{H}_{l, m, s} \otimes \overline{\mathcal{H}}_{l, \bar{m}, \bar{s}}$ coset representations by

$$
\Phi_{m, s ; \bar{m}, \bar{s}}^{l}=\phi_{l, m, s} \otimes \bar{\phi}_{l, \bar{m}, \bar{s}}
$$

Imposing the various constraints, it is then clear that the elliptic genus of these Gepner models is given by [32]

$$
\phi(\tau, z)=\frac{1}{2^{r-1} H} \sum_{a, b=0}^{H} \prod_{i=1}^{r} \sum_{l_{i}=0}^{k_{i}} \sum_{m_{i}=-k_{i}-1}^{k_{i}+2} \mathrm{e}^{\frac{2 \pi \mathrm{i}\left(m_{i}+a\right) b}{k_{i}+2}} I_{m_{i}}^{l_{i}}(\tau, z) I_{m_{i}+2 a}^{l_{i}}(\bar{\tau}, 0) .
$$


Using

$$
I_{m}^{l}(\tau, 0)=\delta_{m, l+1}-\delta_{m,-l-1}
$$

we can directly evaluate (C.16). For the two cases considered in this paper, namely $(1)^{6}$ and $(2)^{4}$, we then find indeed $(2.1)$.

\section{Appendix C.2. D-branes in Gepner models}

A-type (B-type) D-branes satisfy the gluing conditions

$$
\begin{array}{llll}
\left.\left.\left(L_{n}-\bar{L}_{-n}\right) \| \mathrm{A}\right\rangle\right\rangle=0 & \left.\left.\left(J_{n}-\bar{J}_{-n}\right) \| \mathrm{A}\right\rangle\right\rangle=0 & \left.\left.\left(G_{r}^{ \pm}+\mathrm{i} \eta \bar{G}_{-r}^{ \pm}\right) \| \mathrm{A}\right\rangle\right\rangle=0 & \text { (A-type }) \\
\left.\left.\left(L_{n}-\bar{L}_{-n}\right) \| \mathrm{B}\right\rangle\right\rangle=0 & \left.\left.\left(J_{n}+\bar{J}_{-n}\right) \| \mathrm{B}\right\rangle\right\rangle=0 & \left.\left.\left(G_{r}^{ \pm}+\mathrm{i} \eta \bar{G}_{-r}^{ \pm}\right) \| \mathrm{B}\right\rangle\right\rangle=0 & \text { (B-type) }
\end{array}
$$

where $\eta= \pm 1$. In our construction, we shall always consider tensor product A-type D-branes, as well as permutation B-type D-branes. The former are described by the boundary states [33]

$$
\begin{aligned}
\left.\| L_{i}, M_{i}, S_{i}\right\rangle_{\mathrm{A}, s}= & \mathcal{N} \mathrm{e}^{-\frac{-\pi \mathrm{i}}{2} s \sum_{i} S_{i}} \sum_{l_{i}=0}^{k_{i}} \sum_{m_{i}=0}^{k_{i}+1} \sum_{\nu_{i}=0}^{1} \prod_{i}\left(\frac{1+(-1)^{l_{i}+m_{i}+s}}{2}\right) \\
& \times\left(\frac{1}{H} \sum_{t \in \mathbb{Z}_{H}} \mathrm{e}^{2 \pi \mathrm{i} t \sum_{i} \frac{m_{i}}{k_{i}+2}}\right) \cdot(-1)^{\sum_{i} S_{i} \nu_{i}} \mathrm{e}^{\pi \mathrm{i} \sum_{i} M_{i} \frac{m_{i}}{k_{i}+2}} \\
& \left.\times \prod_{i} \frac{S_{L_{i} l_{i}}}{\sqrt{S_{0 l_{i}}}}\left|l_{i}, m_{i}, s+2 \nu_{i}\right\rangle\right\rangle_{\mathrm{A}},
\end{aligned}
$$

where $H=\operatorname{lcm}\left\{k_{i}+2\right\}, \mathcal{N}=\sqrt{H} \prod_{i}\left(\frac{2}{k_{i}+2}\right)^{1 / 4}$ and

$$
L_{i}=0, \ldots, k_{i}, \quad M_{i} \in \mathbb{Z}_{2 k_{i}+4}, \quad S_{i} \in \mathbb{Z}_{4}, \quad L_{i}+M_{i}+S_{i} \text { even, } S_{i}+S_{j} \text { even. }
$$

Here $s=0,1$ labels the NSNS or the RR closed string sector, respectively, and

$$
S_{L_{i} l_{i}}=\sqrt{\frac{2}{k_{i}+2}} \sin \left(\pi \frac{\left(L_{i}+1\right)\left(l_{i}+1\right)}{k_{i}+2}\right)
$$


is the $S$-matrix of the $\mathfrak{s u}(2)$ affine algebra at level $k_{i}$. Furthermore,

$$
\left.\left|l_{i}, m_{i}, s+2 \nu_{i}\right\rangle\right\rangle_{\mathrm{A}} \in \otimes_{i} \mathcal{H}_{\left[l_{i}, m_{i}, s+2 \nu_{i}\right]} \otimes \overline{\mathcal{H}}_{\left[l_{i}, m_{i}, s+2 \nu_{i}\right]}
$$

is the Ishibashi state satisfying the A-type gluing conditions (C.18) for each minimal model factor separately. The left-moving worldsheet fermion number operator acts on this boundary state as

$$
\left.\left.\left.\left.(-1)^{F_{L}} \| L_{i}, M_{i}, S_{i}\right\rangle\right\rangle_{\mathrm{A}, s}=\| L_{i}, M_{i}+1, S_{i}+1\right\rangle\right\rangle_{\mathrm{A}, s}
$$

The overlap between two such boundary states is given by

$$
\begin{aligned}
\mathrm{A}, s & \left.\left\langle L_{i}^{\prime}, M_{i}^{\prime}, S_{i}^{\prime}\left\|q^{\frac{1}{2}\left(L_{0}+\bar{L}_{0}\right)-\frac{c}{24}}(-1)^{F_{L}}\right\| L_{i}, M_{i}, S_{i}\right\rangle\right\rangle_{\mathrm{A}, s} \\
= & \sum_{t \in \mathbb{Z}_{H}} \sum_{\left(l_{i}, m_{i}, s_{i}\right)} \mathrm{e}^{-\frac{\pi \mathrm{i} s}{2} \sum_{i}\left(S_{i}+1-S_{i}^{\prime}+s_{i}\right)} \prod_{i} \delta^{(2)}\left(S_{i}+1-S_{i}^{\prime}+s_{i}\right) \\
& \times \prod_{i}\left[\delta^{\left(k_{i}+2\right)}\left(\frac{M_{i}+1-M_{i}^{\prime}+m_{i}}{2}+t\right) N_{L_{i} l_{i}}^{L_{i}^{\prime}} \chi_{\left[l_{i}, m_{i}, s_{i}\right]}(\tilde{q})\right],
\end{aligned}
$$

where $\tilde{q}$ is the open string parameter.

Given a permutation $\pi \in S_{r}$, a permutation brane $\left.\left.\| \mathrm{B}\right\rangle\right\rangle^{\pi}$ satisfies the $\pi$-twisted B-type boundary conditions

$$
\left.\left.\left.\left(L_{n}^{(i)}-\bar{L}_{-n}^{(\pi(i))}\right) \| B\right\rangle^{\pi}=0, \quad\left(J_{n}^{(i)}+\bar{J}_{-n}^{\pi(i)}\right) \| B\right\rangle\right\rangle^{\pi}=0,
$$

and similarly for the fermionic gluing conditions. For example, for the $(1)^{6}$ model we consider $\pi \in S_{6}$ with cycle decomposition (12)(34)(56). The corresponding permutation brane is defined as [34]

$$
\begin{aligned}
\| L_{1}, & \left.\left.L_{2}, L_{3}, M_{1}, M_{2}, M_{3}, \hat{M}, S_{i}\right\rangle\right\rangle_{\mathrm{B}, s}^{\pi} \\
= & \frac{1}{2^{3}} \frac{1}{\sqrt{3}} \sum_{n \in \mathbb{Z}_{3}} \mathrm{e}^{-\frac{\pi \mathrm{i} i \hat{M}}{3}} \sum_{l_{1}, l_{2}, l_{3}=0}^{1} \sum_{m_{1}, m_{2}, m_{3}=0 \nu_{1}, \ldots, \nu_{6} \in \mathbb{Z}_{2}} \prod_{i}\left(\frac{1+(-1)^{l_{i}+m_{i}+n+s}}{2}\right) \\
& \left.\times \prod_{i} \frac{S_{L_{i} l_{i}}}{S_{0 l_{i}}} \mathrm{e}^{\mathrm{i} \frac{\pi}{3} \sum_{i}^{3} m_{i} M_{i}}(-1)^{\sum_{i}^{6} S_{i} \nu_{i}} \mathrm{e}^{-\mathrm{i} \frac{s \pi}{2} \sum_{i}^{6} S_{i}}\left|\left[l_{i}, m_{i}+n, s+2 \nu_{i}\right]\right\rangle\right\rangle_{\mathrm{B}}^{\pi, g^{n}},
\end{aligned}
$$

where $\hat{M} \in \mathbb{Z}_{6}$, while $L_{i}+M_{i}, \hat{M}+\sum_{i} M_{i}$ and $S_{i}+S_{j}$ are even, so that the boundary state is invariant under $n \mapsto n+3, m_{i} \mapsto m_{i}+3, i=1, \ldots, 3$. 
Here, $\left.\left|\left[l_{i}, m_{i}+n, s_{i}\right]\right\rangle\right\rangle_{\mathrm{B}}^{\pi, g^{n}}$ is the Ishibashi state in the $n$th twisted sector

$\bigotimes_{i=1}^{3}\left(\left(\mathcal{H}_{\left[l_{i}, m_{i}+n, s_{2 i-1}\right]} \otimes \overline{\mathcal{H}}_{\left[l_{i}, m_{i}-n,-s_{2 i}\right]}\right) \otimes\left(\mathcal{H}_{\left[l_{i},-m_{i}+n, s_{2 i}\right]} \otimes \overline{\mathcal{H}}_{\left[l_{i},-m_{i}-n,-s_{2 i-1}\right]}\right)\right)$

that is uniquely characterized (up to normalization) by the B-type boundary conditions (C.24) (together with the corresponding fermionic formulae). Note that

$$
\left.\left.(-1)^{F_{L}} \| L_{i}, M_{i}, \hat{M}, S_{i}\right\rangle_{\mathrm{B}, s}^{\pi}=\| L_{i}, M_{i}, \hat{M}, S_{i}+1\right\rangle_{\mathrm{B}, s}^{\pi}
$$

For the case at hand, the overlap between two such branes with $L_{i}=0$ and $s=1$ (RR sector) is

$$
\begin{aligned}
\stackrel{\pi}{\mathrm{B}, s=1}= & \left\langle\left\langle 0,0,0, M_{1}^{\prime}, M_{2}^{\prime}, M_{3}^{\prime}, \hat{M}^{\prime}, 0 \| q^{\frac{1}{2}\left(L_{0}+\bar{L}_{0}\right)-\frac{c}{24}}(-1)^{F_{L}}\right.\right. \\
& \left.\left.\| 0,0,0, M_{1}, M_{2}, M_{3}, \hat{M}, 0\right\rangle\right\rangle_{\mathrm{B}, s=1}^{\pi} \\
= & \sum_{m_{i}= \pm 1} \mathrm{e}^{-\frac{\pi \mathrm{i}}{2} \sum_{i}^{6}\left(1+m_{i}\right)} \delta^{(3)}\left(\frac{\hat{M}^{\prime}-\hat{M}+\sum_{i} m_{i}}{2}\right) \\
& \times \prod_{i=1}^{3} \delta^{(3)}\left(\frac{M_{i}-M_{i}^{\prime}+m_{2 i-1}-m_{2 i}}{2}\right)
\end{aligned}
$$

In order to determine the relative intersection number between the A-type and the B-type D-branes, we also need to understand the overlap between the different types of branes. For the $(1)^{6}$ model, the only nonvanishing overlaps are between Ishibashi states in the $n=0$ sectors of the form

$$
\bigotimes_{i=1}^{3}\left(\mathcal{H}_{\left[l_{i}, m_{i}, s_{i}\right]} \otimes \overline{\mathcal{H}}_{\left[l_{i}, m_{i}, s_{i}\right]} \otimes \mathcal{H}_{\left[l_{i},-m_{i},-s_{i}\right]} \otimes \overline{\mathcal{H}}_{\left[l_{i},-m_{i},-s_{i}\right]}\right)
$$

Taking into account the implicit relative phase between the Ishibashi states as in [31], one finds that the overlap between the relevant Ishibashi states is

$$
\mathrm{A}\left\langle\left\langle l_{i}, m_{i}, s_{i}\left|q^{\frac{1}{2}\left(L_{0}+\bar{L}_{0}\right)-\frac{c}{24}}\right|\left[l_{i}, m_{i}+n, s_{i}\right]\right\rangle\right]_{\mathrm{B}}^{\pi}=\prod_{i=1}^{3} \mathrm{e}^{\frac{\pi \mathrm{i}}{3} m_{i}-\frac{\pi \mathrm{i}}{2} s_{i}} \chi_{\left[l_{i}, m_{i}, s_{i}\right]}\left(q^{2}\right),
$$


and hence the overlap between the relevant boundary states is given by

$$
\begin{aligned}
\mathrm{A}, s=1 & \left\langle\left\langle 0, M_{i}^{\prime}, 0\left\|q^{\frac{1}{2}\left(L_{0}+\bar{L}_{0}\right)-\frac{c}{24}}(-1)^{F_{L}}\right\| 0,0,0, M_{1}, M_{2}, M_{3}, \hat{M}, 0\right\rangle\right\rangle_{\mathrm{B}, s=1}^{\pi} \\
& =\sum_{m_{1}, m_{2}, m_{3}= \pm 1}-\mathrm{e}^{-\frac{\pi \mathrm{i}}{2}\left(1+\sum_{i} m_{i}\right)} \prod_{i}^{3} \delta^{(3)}\left(\frac{M_{i}-M_{2 i-1}^{\prime}+M_{2 i}^{\prime}+m_{i}+1}{2}\right) .
\end{aligned}
$$

A similar computation can be done for the $(2)^{4}$ model. The B-type permutation brane for $\pi \in S_{4}$ with cycle decomposition (12)(34) is

$$
\begin{aligned}
\left.\left.\| L_{1}, L_{2}, M_{1}, M_{2}, \hat{M}, S_{i}\right\rangle\right\rangle_{\mathrm{B}, s}^{\pi} & \frac{1}{2^{2}} \frac{1}{\sqrt{4}} \sum_{n \in \mathbb{Z}_{4}} \mathrm{e}^{-\mathrm{i} \frac{\pi n}{4} \hat{M}} \sum_{l_{1}, l_{2}=0}^{2} \sum_{m_{1}, m_{2}=0}^{7} \sum_{\nu_{1}, \ldots, \nu_{4}=0}^{1} \prod_{i}\left(\frac{1+(-1)^{l_{i}+m_{i}+n+s}}{2}\right) \\
& \times \prod_{i} \frac{S_{L_{i} l_{i}}}{S_{0 l_{i}}} \mathrm{e}^{\mathrm{i} \frac{\pi}{4} \sum_{i} m_{i} M_{i}}(-1)^{\sum_{i}^{4} S_{i} \nu_{i}} \mathrm{e}^{-\mathrm{i} \frac{\pi s}{2} \sum_{i}^{4} S_{i}}\left|\left[l_{i}, m_{i}+n, s+2 \nu_{i}\right]\right\rangle_{\mathrm{B}}^{\pi, g^{n}},
\end{aligned}
$$

where $\hat{M} \in \mathbb{Z}_{8}$, and $L_{i}+M_{i}, \hat{M}+\sum_{i} M_{i}$ and $S_{i}+S_{j}$ are all even. The leftmoving fermionic number operator acts now by

$$
\left.\left.\left.\left.(-1)^{F_{L}} \| L_{1}, L_{2}, M_{1}, M_{2}, \hat{M}, S_{i}\right\rangle\right\rangle_{\mathrm{B}, s}^{\pi}=\| L_{1}, L_{2}, M_{1}, M_{2}, \hat{M}+4, S_{i}+1\right\rangle\right\rangle_{\mathrm{B}, s}^{\pi},
$$

and the overlap between two branes with $L_{1}, L_{2}=0$ is

$$
\begin{aligned}
\stackrel{\mathrm{B}, s=1}{\pi} & \left\langle\left\langle 0,0, M_{1}^{\prime}, M_{2}^{\prime}, \hat{M}^{\prime}, 0\left\|q^{\frac{1}{2}\left(L_{0}+\bar{L}_{0}\right)-\frac{c}{24}}(-1)^{F_{L}}\right\| 0,0, M_{1}, M_{2}, \hat{M}, 0\right\rangle\right\rangle_{\mathrm{B}, s=1}^{\pi} \\
= & \frac{1}{4} \sum_{\left(l_{i}, m_{i}, s_{i}\right)} \delta_{l_{1} l_{2}} \delta_{l_{3} l_{4}} \prod_{i=1}^{4} \delta^{(2)}\left(s_{i}+1\right) \mathrm{e}^{-\frac{\pi \mathrm{i}}{2} \sum_{i}^{4}\left(1+s_{i}\right)} \\
& \times \delta^{(4)}\left(\frac{\hat{M}^{\prime}-\hat{M}+4+\sum_{i}^{4} m_{i}}{2}\right) \\
& \times \prod_{i=1}^{2} \delta^{(4)}\left(\frac{M_{i}-M_{i}^{\prime}+m_{2 i-1}-m_{2 i}}{2}\right) \chi_{\left[l_{i} m_{i} s_{i}\right]}(\tilde{q})
\end{aligned}
$$




$$
\begin{aligned}
= & \sum_{\substack{m_{1}, m_{2} \in\{1,2,3\} \\
s_{1}, s_{2}= \pm 1}} \mathrm{e}^{-\frac{\pi \mathrm{i}}{2}\left(2+s_{1}+s_{2}\right)} \delta^{(4)}\left(\frac{\hat{M}^{\prime}-\hat{M}+4+\sum_{i}^{2} m_{i}\left(s_{i}+1\right)}{2}\right) \\
& \times \prod_{i=1}^{2} \delta^{(4)}\left(\frac{M_{i}-M_{i}^{\prime}+m_{i}\left(s_{i}-1\right)}{2}\right) .
\end{aligned}
$$

Finally, the RR-overlap between A-type and permutation B-type branes in this model is given by

$$
\begin{aligned}
\mathrm{A}, s=1 & \left\langle\left\langle 0, M_{i}^{\prime}, 0\left\|q^{\frac{1}{2}\left(L_{0}+\bar{L}_{0}\right)-\frac{c}{24}}(-1)^{F_{L}}\right\| 0,0,0, M_{1}, M_{2}, \hat{M}, 0\right\rangle\right\rangle_{\mathrm{B}, s=1}^{\pi} \\
= & \sum_{m_{1}, m_{2}= \pm 1} \mathrm{e}^{-\frac{\pi \mathrm{i}}{2}\left(2+\sum_{i} m_{i}\right)} \delta^{(4)}\left(\frac{M_{1}-M_{1}^{\prime}+M_{2}^{\prime}+m_{1}+1}{2}\right) \\
& \times \delta^{(4)}\left(\frac{M_{2}-M_{3}^{\prime}+M_{4}^{\prime}+m_{2}+1}{2}\right) .
\end{aligned}
$$

\section{References}

[1] T. Eguchi, H. Ooguri and Y. Tachikawa, Notes on the K3 surface and the Mathieu group $M_{24}$, Experiment. Math. 20 (2011), 91, arXiv:hep-th/1004.0956.

[2] T. Gannon, Moonshine beyond the Monster: the bridge connecting algebra, modular forms and physics, Cambridge University Press, Cambridge, UK, 2006.

[3] M. C. N. Cheng, K3 Surfaces, $N=4$ dyons, and the Mathieu group $M_{24}$, Commun. Number Theory Phys. 4 (2010), 623, arXiv:hep-th/1005.5415.

[4] M. R. Gaberdiel, S. Hohenegger and R. Volpato, Mathieu twining characters for K3, J. High Energy Phys. 1009 (2010), 058, arXiv:hep-th/1006.0221.

[5] M. R. Gaberdiel, S. Hohenegger and R. Volpato, Mathieu Moonshine in the elliptic genus of K3, J. High Energy Phys. 10 (2010), 062, arXiv:hep-th/1008.3778.

[6] T. Eguchi and K. Hikami, Note on twisted elliptic genus of K3 surface, Phys. Lett. B694 (2011), 446, arXiv: hep-th/1008.4924.

[7] S. Govindarajan, Brewing moonshine for Mathieu, 2010, arXiv:math.NT/1012.5732. 
[8] S. Govindarajan, BKM Lie superalgebras from counting twisted CHL dyons, 2010, arXiv:hep-th/1006.3472.

[9] S. Govindarajan and K. Gopala Krishna, BKM Lie superalgebras from dyon spectra in $Z(N)$ CHL orbifolds for composite N, J. High Energy Phys. 05 (2010), 014, arXiv:hep-th/0907.1410.

[10] S. Mukai, Finite groups of automorphisms of K3 surfaces and the Mathieu group, Invent. Math. 94 (1988), 183.

[11] S. Kondo, Niemeier lattices, Mathieu groups and finite groups of symplectic automorphisms of K3 surfaces, Duke Math. J. 92 (1998), 593, appendix by S. Mukai.

[12] A. Taormina and K. Wendland, The symmetries of the tetrahedral Kummer surface in the Mathieu group $M_{24}, 2010$, arXiv: hep-th/1008.0954.

[13] P. S. Aspinwall, K3 surfaces and string duality, arXiv:hep-th/9611137.

[14] W. Nahm and K. Wendland, A hiker's guide to K3: Aspects of $N=(4,4)$ superconformal field theory with central charge $c=6$, Commun. Math. Phys. 216 (2001), 85, arXiv:hep-th/9912067.

[15] I. Brunner, M. R. Douglas, A. E. Lawrence and C. Romelsberger, D-branes on the quintic, J. High Energy Phys. 08 (2000), 015, arXiv:hep-th/9906200.

[16] T. Banks and L. J. Dixon, Constraints on string vacua with space-time supersymmetry, Nucl. Phys. B307 (1988), 93.

[17] P. S. Aspinwall, Enhanced gauge symmetries and Calabi-Yau threefolds, Phys. Lett. B371 (1996), 231, arXiv:hep-th/9511171.

[18] A. Strominger, Massless black holes and conifolds in string theory, Nucl. Phys. B451 (1995), 96, arXiv:hep-th/9504090.

[19] J. H. Conway and N. J.A. Sloane, Sphere packings, lattices and groups, in: Grundlehren der Mathematischen Wissenschaften, 290, 3rd edition, Springer-Verlag, New York, 1999.

[20] J. H. Conway, R. T. Curtis, S. P. Norton, R. A. Parker and R. A. Wilson, Atlas of finite groups, Oxford University Press, Oxford, UK, 1985. 
[21] T. Eguchi, H. Ooguri, A. Taormina and S. K. Yang, Superconformal algebras and string compactification on manifolds with $S U(N)$ holonomy, Nucl. Phys. B315 (1989), 193

[22] M. R. Gaberdiel, Lectures on non-BPS Dirichlet branes, Classical Quantum Gravity 17 (2000), 3483, arXiv:hep-th/0005029.

[23] O. Bergman and M. R. Gaberdiel, Non-BPS states in heterotic type IIA duality, J. High Energy Phys. 03 (1999), 013, arXiv:hep-th/9901014.

[24] I. Brunner, R. Entin and C. Romelsberger, D-branes on T(4)/Z(2) and T-duality, J. High Energy Phys. 06 (1999), 016, arXiv:hep-th/9905078.

[25] I. Brunner, M. R. Gaberdiel and C. A. Keller, Matrix factorisations and D-branes on K3, J. High Energy Phys. 06 (2006), 015, arXiv:hep-th/0603196.

[26] R. T. Curtis, On subgroups of ·0. I. Lattice stabilizers, J. Algebra 27 (1973), 549.

[27] I. Brunner, M. R. Gaberdiel, S. Hohenegger and C. A. Keller, Obstructions and lines of marginal stability from the world-sheet, J. High Energy Phys. 05 (2009), 007, arXiv:hep-th/0902.3177.

[28] S. Govindarajan, D. P. Jatkar and K. G. Krishna, BKM superalgebras from counting dyons in $N=4$ supersymmetric type II compactifications, arXiv:hep-th/1106.1318.

[29] V. V. Nikulin, Integer symmetric bilinear forms and some of their geometric applications, Izv. Akad. Nauk SSSR Ser. Mat. 43 (1979), 111.

[30] D. Allcock, Orbits in the Leech lattice, Experiment. Math. 14(4) (2005), 491.

[31] I. Brunner and M. R. Gaberdiel, Matrix factorisations and permutation branes, J. High Energy Phys. 07 (2005), 012, arXiv:hep-th/0503207.

[32] T. Kawai, Y. Yamada and S. K. Yang, Elliptic genera and $N=2$ superconformal field theory, Nucl. Phys. B414 (1994), 191, arXiv:hep-th/9306096.

[33] A. Recknagel and V. Schomerus, D-branes in Gepner models, Nucl. Phys. B531 (1998), 185, arXiv:hep-th/9712186. 
50 Matthias R. Gaberdiel, Stefan Hohenegger and Roberto Volpato

[34] A. Recknagel, Permutation branes, J. High Energy Phys. 04 (2003), 041, arXiv:hep-th/0208119.

Institut FÜr TheORETISChe Physik

ETH ZURICH

CH-8093 ZÜRICH

SWITZERLAND

E-mail address: gaberdiel@itp.phys.ethz.ch

Max-Planck-Institute for Physics

FÖHRINGER RING 6

D-80805 MÜNCHEN

GERMANY

E-mail address: shoheneg@mppmu.mpg.de

Institut FÜR TheOREtische Physik

ETH ZURICH

CH-8093 ZÜRICH

SWITZERLAND

E-mail address: volpato@itp.phys.ethz.ch

ReCeived JuLy 27, 2011 